\title{
Cylindrical integrated optical microresonators: Modeling by 3-D vectorial coupled mode theory
}

\author{
Remco Stoffer, Kirankumar R. Hiremath, Manfred Hammet*, \\ $\mathrm{MESA}^{+}$Institute for Nanotechnology, University of Twente, Enschede, The Netherlands, \\ Ladislav Prkna, Jiří Čtyroký, \\ Institute of Radio Engineering and Electronics, Czech Academy of Sciences, Prague, Czech Republic
}

\begin{abstract}
A spatially three-dimensional, fully vectorial coupled mode theory model for the interaction between several straight or bent dielectric optical waveguides, each supporting multiple modes, is described. The frequency domain model is applied to the coupler regions of cylindrical microresonators, here considered for applications as integrated optical filters. For simple test cases, comparisons with results of beam propagation calculations and of a rigorous system mode analysis provide some validation of the approach. By combination of two coupler representations one obtains a complete 3-D vectorial microresonator description without any free parameters, that permits a convenient investigation of the influence of geometrical parameters on the spectral response. When applied to a microring resonator with pronouncedly hybrid cavity modes, the model reveals the manifold features that may appear in the spectra of these devices. Keywords: integrated optics, numerical modeling, coupled mode theory, bent-straight waveguide couplers, cylindrical microresonators
\end{abstract}

PACS codes: $42.82 .-\mathrm{m}$ 42.82. $\mathrm{Bq}$ 42.82.Et

\section{Introduction}

Over the last decade, microring or -disk resonators for filter applications in integrated optics have been actively studied [1, 2, 3, 4], and technology has come to the point where structures can be reliably fabricated. Composite structures consisting of parallel rings [5] or series of cavity elements [6] have been investigated. If made tunable by thermo-optical or electro-optical means, the microresonators can function as basic building blocks for e.g. modulators, routers, or switches in photonic chips related to optical wavelength-division multiplexing. See e.g. [7] for a recent overview of the field.

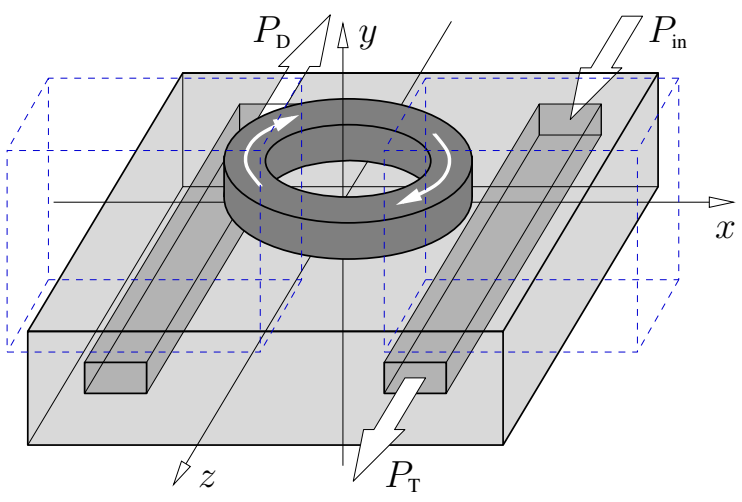

Figure 1: Microring resonator, a 3-D configuration where a circular cavity is placed on top of the two parallel bus waveguides. The optical input power $P_{\text {in }}$ is inserted into one of the bus cores; two output ports through and drop each receive a wavelengthdependent fraction $P_{T}$ and $P_{D}$ of the input power. Two coupler regions (dashed lines) can be identified, where the waves supported by the cavity and bus cores interact.

For this paper we focus on resonator devices that consist of a dielectric ring (or disk) that is evanescently coupled to two straight dielectric waveguides, as shown schematically in Figure 1 The ring and the bus waveguides may be positioned in the same horizontal plane (lateral coupling [8]), or the ring can be located on top of the bus waveguides (vertical coupling [9]). Note that also for vertical coupling, the ring and the straight waveguides can be shifted horizontally with respect to each other. Both lateral and vertical coupling strategies have their own advantages.

* Department of Applied Mathematics, University of Twente Phone: $+31 / 53 / 489-3448$
Fax: $+31 / 53 / 489-4833$
P.O. Box 217, 7500 AE Enschede, The Netherlands

E-mail:m.hammer@math.utwente.nl 
When coupling laterally, one single lithography step is sufficient to define both the bus waveguides and the cavity, allowing for precise alignment control. However, the level of interaction that is needed for the desired resonator performance may require a precisely defined small gap between cavity and bus waveguides, which can be difficult to attain with conventional lithography. This problem can be solved by e.g. employing elliptic cavity shapes, or by adding straight segments to a ring in the coupling section ("racetrack" configuration, [2]). These options, however, have the disadvantage of lowering the minimum bend radius, and thus increasing the intrinsic loss of the cavity when keeping the same circumference, i.e. when keeping the same free spectral range.

In a vertically coupled configuration the vertical gap between the bus waveguides and cavity is defined by means of the deposition step of the buffer material on top of the straight waveguide. The thickness can be controlled with great accuracy, and layers can be made almost arbitrarily thin. Therefore, the coupling strength between the bus waveguides and the cavity can be adjusted very precisely. It is, however, difficult to properly align the cavity exactly with respect to the straight waveguides. Also, imperfect planarization of the buffer material can lead to vertical steps along the light path in the cavity, causing additional scattering loss and possibly reflections at these locations.

In the design of these microresonator structures, it is of vital importance to know the dependence of the coupler performance on the structural parameters. The interaction strength should have the correct magnitude, and should not be too sensitive to the technologically most uncertain parameters (e.g. the horizontal gap for lateral coupling, or the horizontal alignment for vertical coupling). For horizontal coupling, a dimensionality reduction from the full three-dimensional structure to a two-dimensional one can be attempted by means of effective-index like projections methods [1, 2, 10]. Then, two-dimensional simulations can be employed to estimate the bus waveguide-cavity interaction. Among the possible methods are the prominent Finite Difference Time Domain algorithm (FDTD) [11], the application of Helmholtz solvers [12, 13], or modeling in terms of 2-D Coupled Mode Theory (CMT) [14]. Of these, CMT is by far the most efficient method, and has the additional advantage of providing data for the local amplitudes and phases for all the modes that are taken into account. The CMT approach implements directly the physical notions that are usually employed in description of the resonator devices, in contrast to some of the other methods, where an extraction of the complex modal amplitudes is rather difficult, especially if multiple cavity modes are involved.

For the case of vertical coupling, or when effective index methods are simply not accurate enough in the lateral coupling case, fully three-dimensional simulations are required. Additionally, the vectorial nature of the fields may be important; the modes of a bent waveguide or of a straight high-contrast waveguide can be strongly hybrid [15, 16, 17]. In three dimensions, rigorous simulations like FDTD or frequency domain finite element discretizations are almost prohibitively time- and memory-consuming, especially if one wants to perform parameter scans for optimization purposes. So, a more or less rigorous coupled mode theory is a good choice for this problem.

Manifold CMT variants have been proposed, for a variety of different domains of applications [18, 19, 20, 21]. Among these several studies (see e.g. [22, 1, 2, 23, 10, 8]) deal with the evanescent interaction of waves in circularly bent and straight waveguides; these differ in particularly in the way the cavity fields are computed and/or approximated (frequency domain bend modes or time domain gallery resonances), and in the soundness of the heuristics that are applied in the derivation and solution of the coupled mode equations. All of these studies describe spatially 2-D implementations, in some cases applied to refractive index profiles that stem from effective index projections of 3-D structures (exception: coupling to microspheres [23]). A few, rather heuristic, trials for 3-D versions [24, 25] exist as well.

This paper describes an implementation of fully vectorial three-dimensional coupled mode theory, valid in principle for an arbitrary number of (possibly multi-modal) waveguides that can be either straight or circularly bent. The formulation given in Section 2 is based on a variational or reciprocity technique [26, 19] and a study for straight waveguides [27]; it highly resembles the previous 2-D version in Ref. [14]. The outline of the formalism is followed by three examples: Two parallel, straight, high-contrast rectangular cores (Section 3.1), next a vertically-coupled multimodal disk resonator (Section 3.2), and finally a microresonator whose ring supports pronouncedly hybrid modes (Section 3.3). One should emphasize that we are interested here not in an abstract model including fit parameters, but in an effective and accurate tool for ab-initio design.

The CMT approach requires as basis fields solutions of the Maxwell equations for the constituting elementary problems. In case of the 3-D setting these are mode profiles of straight and bend waveguides (or disk profiles) 
with two-dimensional cross sections, the computation of which is in itself a nontrivial and sometimes timeconsuming task. For the present investigation we can profit from a recently presented semianalytical algorithm based on film-mode matching, as described in Refs. [28, 29, 17]. The analytical field representation on a laterally (bend modes: radially) unbounded cross section domain proves to be highly advantageous for the numerical evaluation of the CMT integrals.

\section{Theoretical framework}

In line with the most common ring-resonator model, we assume that the interaction between optical waves in the bus waveguides and the cavity can be restricted to two "coupler regions" around the points where the cores are in closest proximity. In the sketch Figure 1, the dashed lines indicate these regions. Given appropriate modal basis fields of the cavity and of the straight waveguides for a representation of the field outside the couplers, and a suitable description of the coupler performance, it is straightforward to establish a model for the full resonator device. For details we refer to Refs. [30, 21, 31], and to Ref. [14] for the case of multimode cavities that is relevant for the examples in Sections 3.2.2 and 3.3.2

This modeling strategy requires a representation of the cavity-bus-interaction in terms of scattering matrices. Figure 2] shows a top view and cross section of a generic coupler structure, and the enclosure by a rectangular computational window. We aim at a description of the interaction in terms of coupled mode theory, where, with the exception of the additional integrations along the vertical axis, the formalism is basically identical to the 2-D case detailed in Ref. [14]. Still, for completeness the basic equations are outlined below.
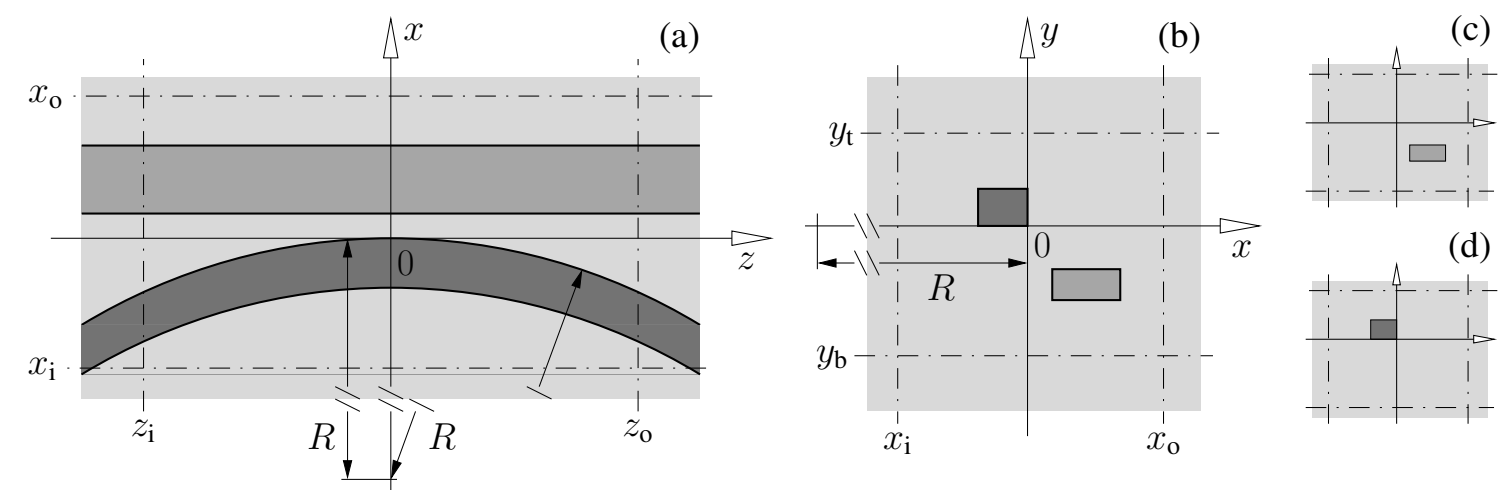

Figure 2: Top view of the coupler region (a) and cross section (b) through the symmetry plane, for a cavity with radius $R$. A rectangular computational window $\left[x_{i}, x_{o}\right] \times\left[y_{b}, y_{t}\right] \times\left[z_{i}, z_{0}\right]$ contains the region where the waves in the cavity and in the bus waveguide interact. The optical field in the full structure (b) is assumed to be well represented by the modal solutions supported by the permittivity distributions of the straight waveguide (c) and the bend (d).

What follows is meant for the frequency domain; all optical waves oscillate with a time dependence $\sim \exp (\mathrm{i} \omega t)$ where the real frequency $\omega=k / \sqrt{\epsilon_{0} \mu_{0}}$ is given in terms of the vacuum wavelength $\lambda=2 \pi / k$, for vacuum wavenumber $k$, permittivity $\epsilon_{0}$, and permeability $\mu_{0}$. We adopt Cartesian coordinates $x, y, z$ as introduced in Figure 2

The coupled mode theory model rests on the assumption that the field solution with electric part $\boldsymbol{E}$ and magnetic part $\boldsymbol{H}$, for the full composite coupler structure as given by the permittivity $\epsilon$, can be adequately represented as a superposition of a number of known electromagnetic fields $\left(\boldsymbol{E}_{m}, \boldsymbol{H}_{m}\right)$ that are valid solutions of the Maxwell equations for problems specified by permittivity profiles $\epsilon_{m}$. In general the choice of the constituting structures, and the selection of basis fields that are supported by these, is a matter of physical intuition, and usually also of convenience. The obvious guideline (cf. Eq. (5)) is that the differences $\left|\epsilon-\epsilon_{m}\right|$ between the permittivities of the full and of the constituting structures are as small as possible, while it is still convenient to compute the required basis solutions. Note that in general for each constituting permittivity profile several modal solutions exist, that can be relevant for the field in the full structure. To avoid notational overhead, here we assume that a discrete set of basis fields $\left(\boldsymbol{E}_{m}, \boldsymbol{H}_{m}\right)$ related to permittivities $\epsilon_{m}$ is known, where the constituting profiles $\epsilon_{m}$ are not necessarily distinct.

For the coupler of Figure 2, a natural division is hinted at by the sketches (c) and (d). One expects that the field in the full structure with cross section Figure 2(b) can be described properly by bend modes supported by the 
ring cavity (Figure 2]d), a segment of the ring core in an otherwise homogeneous cladding), superimposed with guided modes of only the straight waveguide (Figure 2/c), the straight core and cladding, for an absent ring). For the present discussion we restrict the basis fields to unidirectional mode propagation in all guiding regions, as indicated by the arrows in Figure 1. Reflections of optical waves in the coupler regions are thus neglected. The approximation can be more or less appropriate for a specific structure; unfortunately, this can not be checked directly within the coupled mode formalism. For the devices considered in Section 3 however, we expect only minor contributions of reflected waves, due to the relatively large cavity radii with longitudinally smoothly varying permittivity profiles in the coupler regions. According to the comparison with rigorous numerical simulations (2-D) in Ref. [14], disregarding reflections can be an adequate approximation even for quite small, high contrast cavities, at least what concerns the spectral power transmission.

Then for the coupled mode approach one assumes that the optical field of the complete structure can be approximated well by a linear combination of the basis fields, each modulated by an amplitude function that depends on only one variable, where the choice of this evolution variable is rather arbitrary. Obvious options for the present bent-straight waveguide coupler are either the Cartesian coordinate $z$ along the axis of the straight core, or the angular coordinate of the cylindrical system related to the circular cavity. As the more convenient alternative we opt for the Cartesian $z$-coordinate as the evolution variable for all basis fields, such that the ansatz for the complete field reads:

$$
\boldsymbol{E}(x, y, z)=\sum_{m} A_{m}(z) \boldsymbol{E}_{m}(x, y, z), \quad \boldsymbol{H}(x, y, z)=\sum_{m} A_{m}(z) \boldsymbol{H}_{m}(x, y, z) .
$$

Here the terms $\boldsymbol{E}_{m}(x, y, z)$ and $\boldsymbol{H}_{m}(x, y, z)$ consist of the mode profiles, multiplied by the appropriate exponential dependences on the respective propagation coordinate. In case of the bend modes used for the representation of the field in the cavity, the full basis fields (vectorial mode profile and the dependence on the angular coordinate) have to be transformed from their natural cylindrical coordinate system to the Cartesian one; cf. the 2-D case [14] for details of the ansatz. We assume that all basis fields are power-normalized, i.e. the $z$-component (modes of the straight waveguide) or the angular component (bend modes of the cavity) of the Poynting vector associated with the respective mode profiles evaluates to unity. Note that even quite radiative bend modes decay sufficiently fast for large radial coordinates [32]; problems due to non-convergent integrals do not arise.

To derive evolution equations for the amplitudes $A_{m}$, we make use of Lorentz' reciprocity theorem [19], which is a direct consequence of the Maxwell equations. It states the following equality for solutions $\left(\boldsymbol{E}_{p}, \boldsymbol{H}_{p}\right)$ and $\left(\boldsymbol{E}_{q}, \boldsymbol{H}_{q}\right)$ of structures with (real) permittivity distributions $\epsilon_{p}$ and $\epsilon_{q}$, respectively:

$$
\nabla \cdot\left(\boldsymbol{E}_{p} \times \boldsymbol{H}_{q}^{*}+\boldsymbol{E}_{q}^{*} \times \boldsymbol{H}_{p}\right)=-\mathrm{i} \omega \epsilon_{0}\left(\epsilon_{p}-\epsilon_{q}\right) \boldsymbol{E}_{p} \cdot \boldsymbol{E}_{q}^{*} .
$$

By applying Eq. (2) to the composite fields (11) with the full permittivity $\epsilon$ on the one hand, and to one of the basis fields $\boldsymbol{E}_{k}, \boldsymbol{H}_{k}$ with the related constituting permittivity $\epsilon_{k}$ on the other hand, one obtains, after integrating over the $x$-y-cross-section plane

$$
\iint \nabla \cdot \sum_{m}\left(A_{m} \boldsymbol{E}_{m} \times \boldsymbol{H}_{k}^{*}+\boldsymbol{E}_{k}^{*} \times A_{m} \boldsymbol{H}_{m}\right) \mathrm{d} x \mathrm{~d} y=-\mathrm{i} \omega \epsilon_{0} \iint \sum_{m} A_{m}\left(\epsilon-\epsilon_{k}\right) \boldsymbol{E}_{m} \cdot \boldsymbol{E}_{k}^{*} \mathrm{~d} x \mathrm{~d} y .
$$

Since the amplitudes $A_{m}$ depend only on the evolution coordinate $z$, this can be rewritten as

$$
\begin{aligned}
& \sum_{m} \frac{\mathrm{d} A_{m}}{\mathrm{~d} z} \iint \boldsymbol{e}_{z} \cdot\left(\boldsymbol{E}_{m} \times \boldsymbol{H}_{k}^{*}+\boldsymbol{E}_{k}^{*} \times \boldsymbol{H}_{m}\right) \mathrm{d} x \mathrm{~d} y \\
+ & \sum_{m} A_{m} \iint \nabla \cdot\left(\boldsymbol{E}_{m} \times \boldsymbol{H}_{k}^{*}+\boldsymbol{E}_{k}^{*} \times \boldsymbol{H}_{m}\right) \mathrm{d} x \mathrm{~d} y=-\mathrm{i} \omega \epsilon_{0} \sum_{m} A_{m} \iint\left(\epsilon-\epsilon_{k}\right) \boldsymbol{E}_{m} \cdot \boldsymbol{E}_{k}^{*} \mathrm{~d} x \mathrm{~d} y,
\end{aligned}
$$

where $\boldsymbol{e}_{z}$ is the unit vector in the $z$-direction. In the second summation, one recognizes the left-hand side of Lorentz' reciprocity theorem (2). The substitution simplifies the equation as

$$
\sum_{m} \frac{\mathrm{d} A_{m}}{\mathrm{~d} z} \iint \boldsymbol{e}_{z} \cdot\left(\boldsymbol{E}_{m} \times \boldsymbol{H}_{k}^{*}+\boldsymbol{E}_{k}^{*} \times \boldsymbol{H}_{m}\right) \mathrm{d} x \mathrm{~d} y=-\mathrm{i} \omega \epsilon_{0} \sum_{m} A_{m} \iint\left(\epsilon-\epsilon_{m}\right) \boldsymbol{E}_{m} \cdot \boldsymbol{E}_{k}^{*} \mathrm{~d} x \mathrm{~d} y
$$


Upon combining the amplitudes $A_{m}$ into a single vector $\boldsymbol{A}$, we end up with the matrix equation

$$
\mathrm{O}(z) \frac{\mathrm{d} \boldsymbol{A}}{\mathrm{d} z}(z)=\mathrm{C}(z) \boldsymbol{A}(z)
$$

with $z$-dependent (power-) coupling matrices $\mathrm{O}$ and $\mathrm{C}$, whose entries are given by the integrals on the left and right hand sides of Eq. (5). By numerical evaluation the system of equations (5) or (6) is finally solved for the unknown mode amplitudes. The procedure consists of the numerical integration of the differential equation along the $z$-interval $\left[z_{\mathrm{i}}, z_{\mathrm{o}}\right]$ that represents the coupler region, with repeated numerical quadrature of the local integrals over a suitable transverse computational window $\left[x_{\mathrm{i}}, x_{\mathrm{o}}\right] \times\left[y_{\mathrm{b}}, y_{\mathrm{t}}\right]$ (where in particular a large interval along the $x$-direction can be required, if highly radiative bend mode profiles are involved). Due to the linearity of the system, this procedure can be formulated directly for the transfer matrix that relates the amplitudes $\boldsymbol{A}\left(z_{\mathrm{o}}\right)$ in the output plane of the coupler to the amplitudes $\boldsymbol{A}\left(z_{\mathrm{i}}\right)$ in the input plane.

According to Refs. [33, 14], for specific structures the elements of the resulting transfer matrix that correspond to the power transfer to the straight waveguide exhibit a rather oscillatory behaviour with respect to the extension $z_{\mathrm{o}}$ of the longitudinal computational interval, even for $z$-positions beyond the region where one can expect the interaction to be significant. These oscillations are caused by non-negligible strengths of the radiating fields from the cavity, in the output plane of the coupler and outside. One can extract the proper stationary amplitudes of the straight waveguide modes, even for a seemingly too small computational $z$-interval, by projecting the full field Eq. (11) at $z=z_{\mathrm{o}}$ onto the respective basis mode profiles ("taking overlap integrals"). See Refs. [33, 14] for details of this procedure. Oscillations of this kind are not observed for the transfer matrix entries related to the power transfer to the bend modes.

Finally, by incorporating the straight mode projections into the transfer matrix, one obtains the required scattering matrix $S$ for the coupler regions. In the following sections we adopt a symbolic notation for the modal basis fields to identify the entries $\mathrm{S}_{o i}$ of the scattering matrix. The element $\mathrm{S}_{o i}$ ("coupling coefficient") corresponds to the interaction between input mode $i$ and output mode $o$, where, for properly power-normalized basis modes, the absolute square $\left|\mathrm{S}_{o i}\right|^{2}$ of this quantity can be interpreted as the relative power transferred to mode $o$, given a single unit input in mode $i$.

Once the coupler scattering matrices and the propagation constants for the cavity modes are available, evaluation of the power transfer of the full microresonator device is straightforward. We apply the equations for multimode configurations given in Ref. [14]. All results in Sections 3.2.2] and 3.3.2] are meant for arrangements with two parallel bus waveguides and identical coupler regions, i.e. structures with twofold symmetry with respect to the planes $x=0$ and $z=0$ in Figure 1 An analogous treatment can be applied to cavities coupled to a single bus waveguide, or to non-symmetric two-bus configurations, with e.g. different gaps in the two coupler regions, due to a misalignment of the cavity during fabrication [14].

In principle, for calculating the spectral response of the microresonator one needs to repeat the entire procedure (mode analysis, CMT integration, evaluation of the resonator power transfer) for a sequence of wavelengths. However, if only a narrow wavelength range is of interest, one can expect that the coupler scattering matrices are almost constant over that range, and that the resonances in the cavity loop originate from an also only moderate variation of the propagation constants of the cavity modes with the vacuum wavelength. Consequently, the spectral results in Section 3 are evaluated by calculating the coupler scattering matrices once for a central wavelength, and by employing a linear (interpolated) wavelength dependence of the propagation constants of the cavity modes, where suitable correction terms take into account that substantial parts of the cavity are included into the coupler scattering matrices. Explicit expressions are given in [14]; we observed this to be an excellent approximation. If necessary, an easy way to improve the spectrum evaluation would be to employ higher order functions for the complex scattering matrices and the effective indices of the cavity modes, in each case determined as interpolations through a few complete CMT calculations for different wavelengths.

\section{Numerical results}

The basis fields for the CMT computations in this section are generated by a rigorous, fully vectorial mode solver for straight and bent dielectric optical waveguides, based on the film-mode-matching (FMM) method. A modal eigenvalue problem is tackled semi-analytically by dividing the waveguide cross section plane into 
vertical slices, such that the permittivity profile is constant along the horizontal / radial axis. Separately on each slice, the modal field is expanded into eigenfunctions (modes of 1-D multilayer slab waveguides) associated with the respective local refractive index profile, where the sets of eigenfunctions are discretized by employing Dirichlet boundary conditions on horizontal planes suitably far above and below the interesting region around the waveguide core. The 3-D modes are found where the expansions on the individual slices can be connected such that the full field satisfies the continuity requirements at the vertical slice interfaces, and shows the appropriate behaviour in the outer slices. While the algorithmic details can be found elsewhere [28, 29, 17], for completeness we mention the few numerical parameters that enter the mode analysis procedures. These are the numbers of slab modes $M_{\mathrm{s}}$ per slice and polarization direction, and the vertical computational window $\left[y_{\mathrm{b}}^{\mathrm{M}}, y_{\mathrm{t}}^{\mathrm{M}}\right]$.

Once the basis fields are at hand, the CMT model requires numerical procedures at two other places, i.e. for the repeated evaluation of the integrals in Eq. (5) over the transverse computational window (so far only a simple trapezoidal rule is applied, though with proper positioning of the meshpoints for the quadrature of the discontinuous, piecewise smooth integrands, and with duplicated nodal values at discontinuities), and for the integration of the system of ordinary differential equations Eq. (6) (here we implemented a Runge-Kutta scheme of order four [34]). Apart from the respective extensions of the computational window, the stepsizes $\Delta x, \Delta y$ for the summation of the transverse integrals, and $\Delta z$ for the solution of the ordinary differential equation enter as numerical parameters. Note that the basis fields for the CMT analysis are given quasi-analytically; the spatial discretizations do not influence the computation of mode profiles and propagation constants.

\subsection{Straight waveguide coupling}

As a test of the CMT implementation, we consider briefly an example of two parallel straight waveguides. Identical programs as for the bent-straight waveguide couplers in Sections 3.2.1 and 3.3.1 are applied. Figure 3 introduces the coupler cross section which consists of two silicon-nitride cores on top of a silicon dioxide substrate, clad with a polymer material.

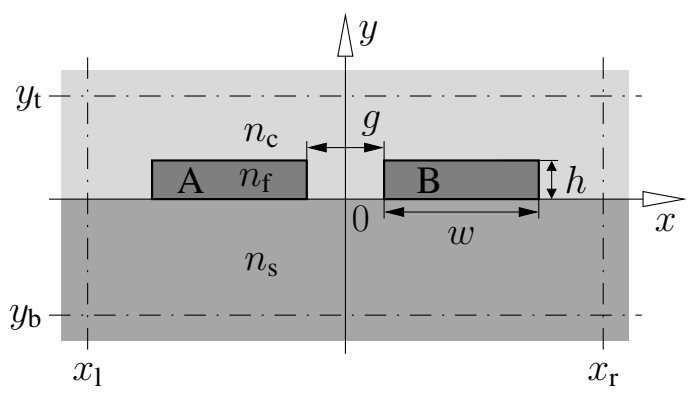

Figure 3: Two parallel straight waveguides, identical cores $A, B$ of height $h=0.185 \mu \mathrm{m}$, width $w=3.15 \mu \mathrm{m}$, and refractive index $n_{f}=1.995$, on a substrate with refractive index $n_{s}=1.45$ and covered by a material with index $n_{c}=1.401$. Configurations with varying gap $g$ are considered, for TM polarized light with wavelength $\lambda=1.55 \mu \mathrm{m}$. Numerical parameters, calculation of basis modes: $M_{s}=120,\left[y_{b}^{M}, y_{t}^{M}\right]=[-4,4] \mu \mathrm{m}$; integration of the CMT equations: $\Delta x=50 \mathrm{~nm}, \Delta y=37 \mathrm{~nm}, \Delta z=2.0 \mu \mathrm{m}$; $\left[x_{1}, x_{r}\right]=[-8,8] \mu \mathrm{m},\left[y_{b}, y_{t}\right]=[-3.7,3.7] \mu \mathrm{m},\left[z_{i}, z_{o}\right]=[0, L]$.

The basis modes for the CMT simulation are computed for constituting permittivity profiles that contain only one of the two cores, where we restrict the basis set to the two vectorial, fundamental TM-like modes. Figure 4 shows the scattering matrix elements of the coupler, calculated for a core separation $g=50 \mathrm{~nm}$, for coupler segments of varying length $L$. Apart from issues related to the projections at the input and output planes, one can interpret the curves as the evolution of the CMT amplitudes: For a normalized input in waveguide $i$ at $z=z_{\mathrm{i}}=0$, the scattering matrix coefficient $\mathrm{S}_{o i}(L)=A_{o}(z)$ gives the local amplitude of mode $o$ at $z=z_{\mathrm{o}}=L$. Obviously the implementation respects exactly the symmetry of the problem with respect to the plane $x=0$.

Alternatively, one may view the two cores as a single composite waveguide. For this system, traditional mode solvers can be employed to compute the two lowest order symmetric and antisymmetric TM modes, with (nearly degenerate) propagation constants $\beta_{\mathrm{s}}$ and $\beta_{\mathrm{a}}$, respectively. The beating of these two modes as they propagate with equal initial amplitudes results in a pattern that also corresponds to a power transfer between the two waveguides. Apart from issues related to the projection onto the modes of the single cores, for a unit input into core $\mathrm{A}$ at $z=0$, the power in core $\mathrm{A}$ at $z=L$ is given by the expression $\left|S_{\mathrm{AAA}}\right|^{2}=\cos ^{2}\left(\left(\beta_{\mathrm{s}}-\beta_{\mathrm{a}}\right) L / 2\right)$. Figure 5 compares results calculated by coupled mode theory and by the system mode analysis, for varying coupler gap $g$ and a fixed length $L$ of the coupling segment. One observes a good agreement apart from very narrow gap widths, where apparently the system modes of the structure can no longer be approximated well by just an even and odd combination of modes of the individual waveguides (the spatial discretization for the CMT 


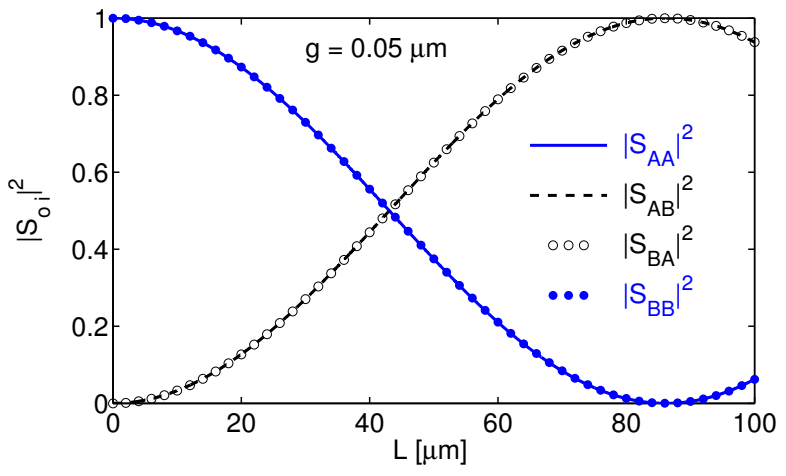

Figure 4: Scattering matrix elements for the straightwaveguide coupler of Figure 3 for different lengths $L$ of the coupling segment. A coefficient $\left|S_{o}\right|^{2}$ can be regarded as the output power observed in channel $o$, given a unit input in channel $i$, for $o, i=A, B$.

integration may appear rather coarse, see the caption of Figure 3, note, however, that the waveguide dimensions are captured precisely by the positioning of the nodal points, while the fields are smooth and relatively slowly varying in between the discontinuities). In order to provide a completely independent test, here we applied the modesolver embedded in a commercial simulation package [35] for the system mode computations.

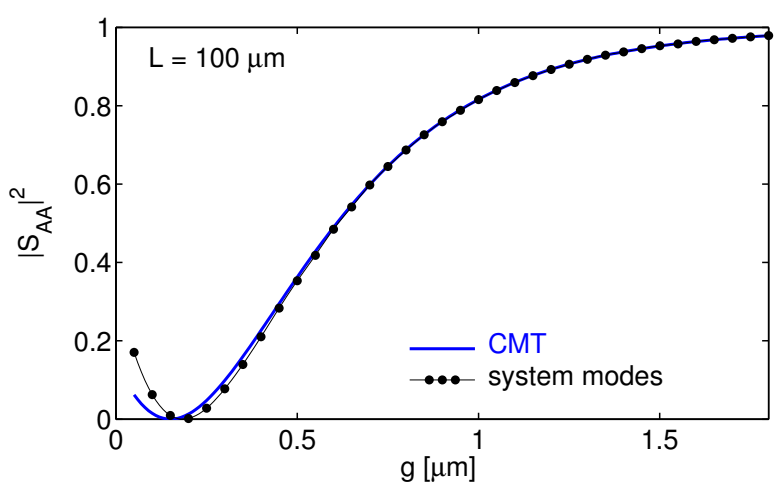

Figure 5: Throughput power $\left|\mathrm{S}_{A A}\right|^{2}$ for a coupler segment according to Figure 3 of length $L=100 \mu \mathrm{m}$, versus the core separation g. CMT results (bold line) are compared with rigorous system mode analysis (circles).

\subsection{Vertical coupling between straight waveguide and disk}

Figure @introduces a coupler configuration that combines a straight silicon-nitride / silicon-dioxide waveguide, and a polymer disk, clad with a polymer material, in a vertically coupled resonator structure. Horizontal and vertical variations of the relative positions of bus waveguide and cavity will be considered.

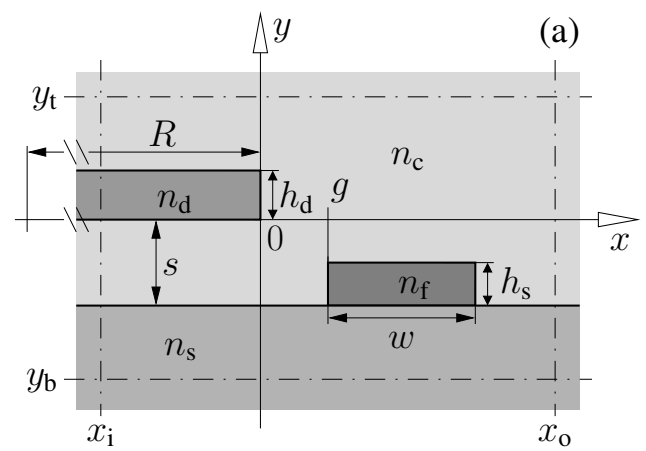

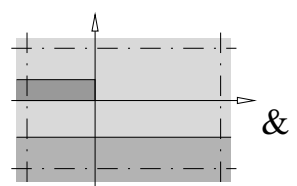

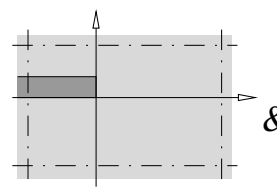

(b)

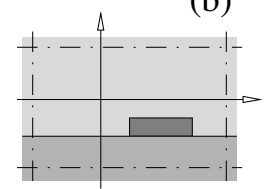

(c)

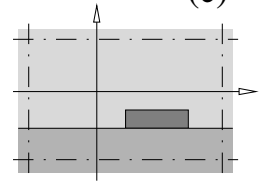

Figure 6: (a): Straight-to-disk coupler configuration, cross section at the center $z=0$ of the coupler region. The straight core of width $w=2.0 \mu \mathrm{m}$, height $h_{s}=140 \mathrm{~nm}$, and refractive index $n_{f}=1.98$ is deposited on a substrate with index $n_{s}=1.45$ and covered by a material with refractive index $n_{\mathfrak{c}}=1.4017$. The relative position of the polymer disk with refractive index $n_{d}=1.6062$, height $h_{d}=1.0 \mu \mathrm{m}$, and radius $R=100 \mu \mathrm{m}$ is defined by the distance from the substrate $s$ and the gap $g$ between the disk rim and the left flank of the straight core (where a negative $g$ indicates overlapping components). The modal analysis and all coupler simulations are meant for a vacuum wavelength $\lambda=1.55 \mu \mathrm{m}$. The $C M T$ equations are integrated with numerical parameters $\Delta x=40 \mathrm{~nm}, \Delta y=20 \mathrm{~nm}, \Delta z=2.0 \mu \mathrm{m} ;\left[x_{i}, x_{o}\right]=[-12,4] \mu \mathrm{m}$, $\left[y_{b}, y_{t}\right]=[-4 \mu \mathrm{m}-s, 4 \mu \mathrm{m}-s],\left[z_{i}, z_{o}\right]=[-30,30] \mu \mathrm{m}$. (b), (c): Two possible choices of permittivity profiles for the computation of CMT basis fields. The disk modes can be computed for a structure that includes (b) or excludes the substrate (c). 


\subsubsection{Coupler simulations}

The coupled mode simulations start with the definition of the constituting profiles for the computation of the CMT basis fields. The two most obvious choices are indicated by parts (b) and (c) of Figure 6 For a substrate slab that extends far enough in the $x-z$ plane, both options lead to a rotationally invariant permittivity profile, as required for the computation of the cavity modes by the bend mode solver. In setting (b), however, the constituting profile for the disk modes is closer to the full permittivity than in (c), hence one expects a better approximation to the total field in (b) than in (c). The presence of the silicon dioxide substrate may significantly influence the disk mode profiles and propagation constants; with its refractive index above the level of the cladding, the substrate effects a preferred downward direction of the radiative parts of the cavity mode profiles. This effect is taken into account when the substrate is included into the basis mode calculations. Furthermore, in setting (b) the integrals on the right hand side of Eq. (5) extend only over the disk and straight core regions, not over the substrate domain. Therefore we opt for setting (b) first. Option (c) would have the advantage that the calculation of the bend basis fields is simplified, and that in the CMT simulations also the vertical distance $s$ can be varied without the necessity to recalculate the disk mode profiles; a comparison between the two settings follows at the end of this section.

\subsubsection{Basis modes}

This particular disk structure supports multiple modes with relatively low levels of attenuation, the three lowest order ones will be taken into account in the following simulations. We restrict the basis set to TE-like fields in this example, since the hybridness of the modes is negligible and different polarizations are thus not expected to interact significantly. Table 1 lists the (complex) effective indices of the three disk modes for vertical separations $s$ of 0.5 and $1.0 \mu \mathrm{m}$; Figure 7 illustrates the bend mode profiles for the smaller separation $s=0.5 \mu \mathrm{m}$.

\begin{tabular}{l|l|l} 
& $s=0.5 \mu \mathrm{m}$ & $s=1.0 \mu \mathrm{m}$ \\
\hline $\mathrm{TE}_{0}$ & $1.503778-\mathrm{i} 1.35 \cdot 10^{-9}$ & $1.503450-\mathrm{i} 1.53 \cdot 10^{-9}$ \\
$\mathrm{TE}_{1}$ & $1.474931-\mathrm{i} 1.77 \cdot 10^{-6}$ & $1.474585-\mathrm{i} 4.96 \cdot 10^{-7}$ \\
$\mathrm{TE}_{2}$ & $1.451487-\mathrm{i} 5.05 \cdot 10^{-5}$ & $1.451093-\mathrm{i} 1.56 \cdot 10^{-5}$
\end{tabular}

Table 1: Effective indices $n_{\text {eff }}$ of the modes of the disk in Figure 6 related to the disk rim, i.e. the modal fields evolve with the angular coordinate $\theta$ in the cylindrical coordinate system of the disk as $\sim \exp \left(-\mathrm{i} k n_{\mathrm{eff}} R \theta\right)$, where $R$ is the radius of the disk rim. The straight waveguide supports one guided TE-like mode with an effective refractive index of 1.48229.
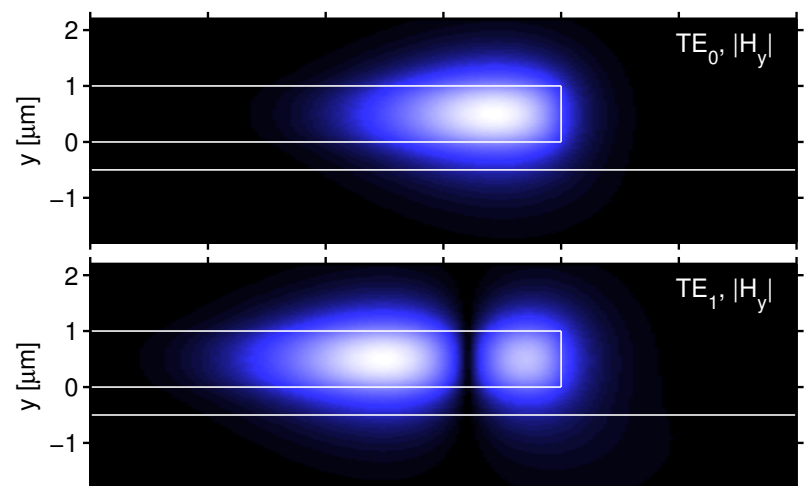

Figure 7: Profiles of the three lowest order modes supported by the disk in Figure 6 (b), for a separation $s=$ $0.5 \mu \mathrm{m}$. The plots show the absolute value $\left|H_{y}\right|$ of the

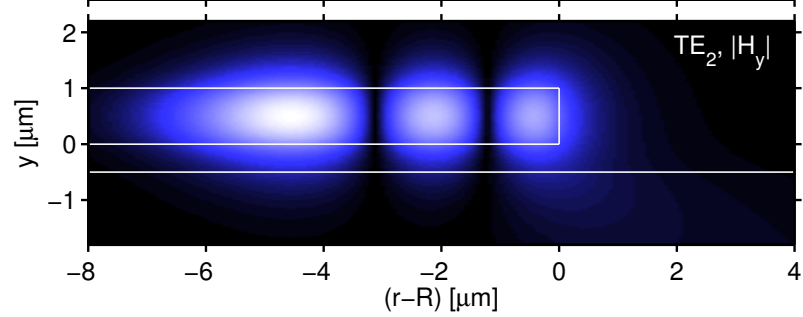
dominant magnetic field component for the TE-like modes. $r$ denotes the radial coordinate in a cylindrical coordinate system with origin at the center of the disk; hence, for the vertical symmetry plane of the coupler, $r-R$ coincides with the $x$-coordinate as introduced in Figures 2 and $[\mathrm{Nu}$ merical parameters for the mode analysis are $M_{s}=200$, $\left[y_{b}^{M}, y_{t}^{M}\right]=[-10 \mu m-s, 7 \mu m-s]$.

Just as in the planar case [32], for the vertically single-mode disk the mode profiles are distinguished by a growing number of radial minima, here in the dominant magnetic field component. Also similar to the planar configurations, for growing mode order one observes a decrease of the real part of the effective mode indices, and an increase of the mode attenuation. The distance of the substrate has only a moderate influence, where 
(with the exception of the negligible, perhaps slightly inaccurate levels of the fundamental field) the attenuations are higher for the smaller separation (additional "leakage" into the substrate).

\subsubsection{Coupler scattering matrices}

With a modal basis set that includes all four fields (i.e. the three disk modes of Figure 7 together with the fundamental TE-like mode of the bus waveguide), the CMT simulations generate scattering matrices of a size $4 \times 4$. Figure 8 summarizes the dependence of the 16 coefficients on the relative horizontal position of bus waveguide and cavity, for two different vertical separations. A value $g=0$ indicates a setting where, in a top view as in Figure 2, the inner flank of the bus waveguide just touches the rim of the cavity disk.
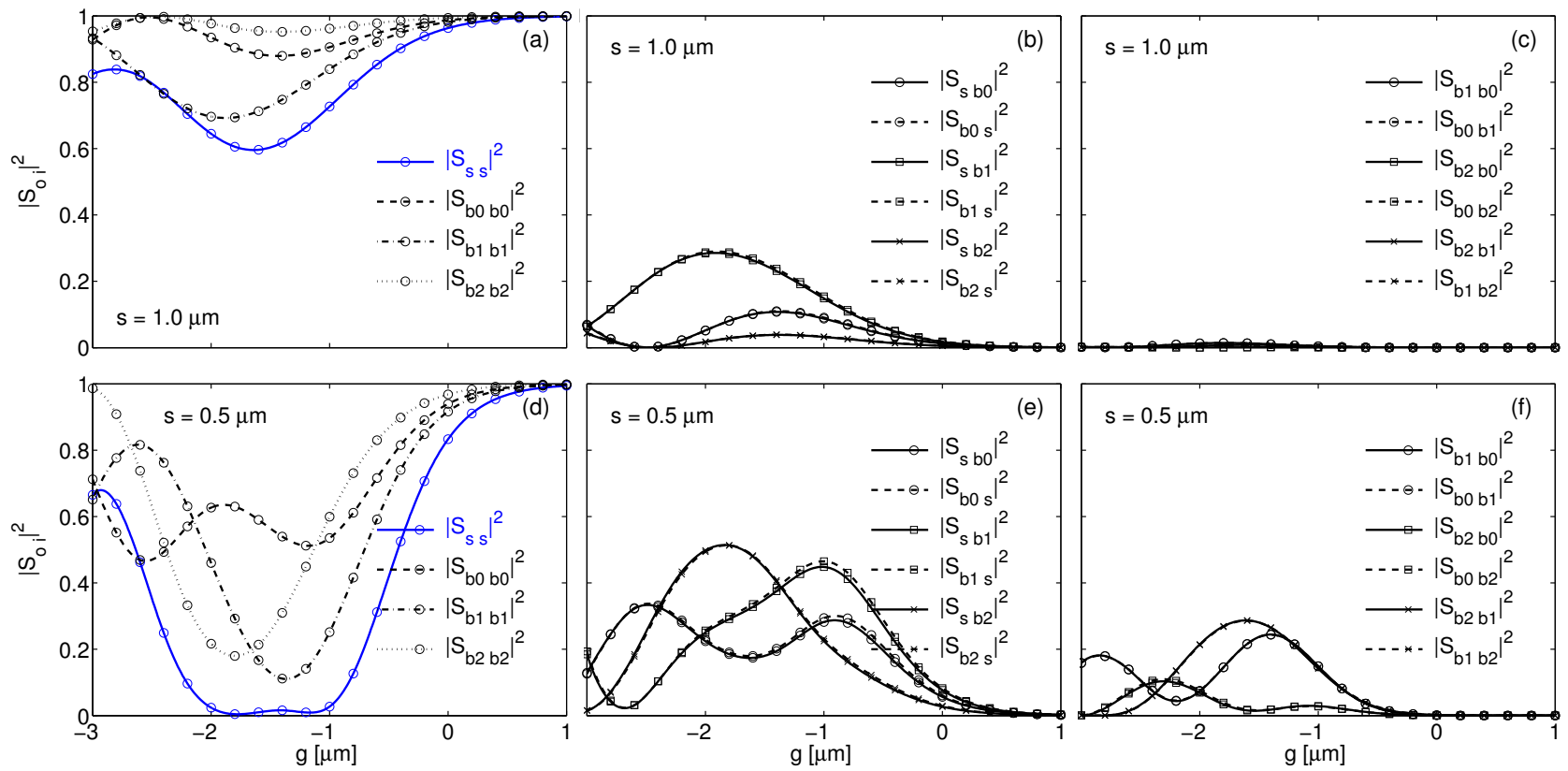

Figure 8: Scattering matrix coefficients for the structure of Figure 6 for vertical separations $s=1.0 \mu \mathrm{m}$ (top row) and $s=0.5 \mu \mathrm{m}$ (bottom row) versus the lateral position $g$ of the disk rim relative to the left flank of the straight waveguide. (a, $d)$ : the self-coupling power of each mode, $(b$, e) the cross-coupling powers between the mode of the straight waveguide and the bend fields, $(c, f)$ : the cross-coupling coefficients for the bend modes. CMT calculations that take three bend modes into account.

A symbolic notation is applied to associate the scattering matrix coefficients with the basis modes: Subscripts s, b0, b1, and b2 indicate the TE-like modes of the straight waveguide and the three bend modes of the cavity (with growing mode order), respectively. An effort to explain these interaction levels based on the properties of the basis modes and their respective geometrical positions turns out to be quite involved. In any case it is necessary to distinguish clearly between the local strength of the "coupling process" (i.e. the rapidity of changes in the evolution of the CMT amplitudes $\boldsymbol{A}(z)$, not shown here), and the net effect of the coupler, given by the scattering matrix elements for a fixed computational window. Only this net effect is relevant for the resonator performance, and discussed in the following paragraphs.

With respect to the vertical distance $s$, as to be expected the interaction is much stronger for the smaller separation. This becomes most evident by a comparison of plots (c) and (f), where for $s=0.5 \mu \mathrm{m}$ in (f) the stronger perturbation by the closer bus core effects substantially larger cross-coupling of the - otherwise uncoupled bend modes than for the larger distance $s=1.0 \mu \mathrm{m}$ in (c). What concerns the lateral bus/cavity position and the role of the individual modes, although there is an obvious influence of the locations of the mode intensity maxima, the bend mode maxima overlap with the straight mode fields on only part of the interval $\left[z_{\mathrm{i}}, z_{\mathrm{o}}\right]$ due to the curvature of the disk. Also the familiar "phase matching argument" can not be applied directly, due to the arbitrariness in the definition of the effective indices of the bend modes (still, e.g. for $s=1.0 \mu \mathrm{m}$ one finds the strongest interaction of the straight waveguide mode $s$ with the first order bend mode b1 which differs the least from s in effective index). We retreat with the remark that the CMT implements precisely these physical arguments, and use the results for the scattering matrices for an interpretation of the related resonator spectra in Section 3.2.2 
With the computational interval $\left[z_{\mathrm{i}}, z_{\mathrm{o}}\right]$ as given in the caption of Figure 6 , the present coupler structure is symmetric with respect to the plane $z=0$. Assuming that also the individual cavity bend modes constitute "decoupled" input / output "ports" (suitable orthogonality properties hold at least for 2-D bend modes [32]), reciprocity arguments as outlined in Refs. [19, 31] can be applied, that predict a symmetric scattering matrix. The excellent coincidence of the dashed and continuous lines in Figure 8 (b, c, e, f) indicates that the present CMT implementation satisfies that requirement remarkably well (in contrast e.g. to Ref. [25], where the authors claim to have computed "asymmetric coupling" effects). For each pair of modes $m$ and $n$, one finds the power $\left|\mathrm{S}_{n m}\right|^{2}$ transferred from mode $m$ at $z=-z_{\mathrm{o}}$ to mode $n$ at $z=z_{\mathrm{o}}$ to be equal to the power transfer $\left|\mathrm{S}_{m n}\right|^{2}$ from mode $n$ at $z=-z_{\mathrm{o}}$ to mode $m$ at $z=z_{\mathrm{o}}$, irrespectively whether this concerns different cores or lossy modes.

\subsubsection{Role of individual modes}

Figure 9 allows to examine the relevance of the individual basis modes and their interaction for the CMT results, for the weaker (a) and stronger interaction (b) at $s=1.0 \mu \mathrm{m}$ and $s=0.5 \mu \mathrm{m}$, respectively, where the plots are restricted to the throughput power $\left|\mathrm{S}_{\mathrm{ss}}\right|^{2}$ of the bus waveguide. The bold lines correspond to CMT simulations with fewer basis modes. If, in addition to the field $s$ of the straight waveguide, only the fundamental bend mode b0 is taken into account, the results s, b0 look very much like the familiar power transfer through an imperfectly phase-matched ordinary directional coupler: A certain power fraction couples from the straight waveguide to the disk, but when - here with decreasing $g$ - the coupling strength reaches a certain level, all power is transferred back from the disk into the straight core. This behaviour is observed for both separations in (a) and (b). When additional bend modes are taken into account, however, this full back-coupling does no longer happen. There still are variations of the transmitted power, but the curves do not reach unity anymore. This can be explained by the different phase velocities of the bend modes, and by the interaction between the bend modes that is effected by the straight core.
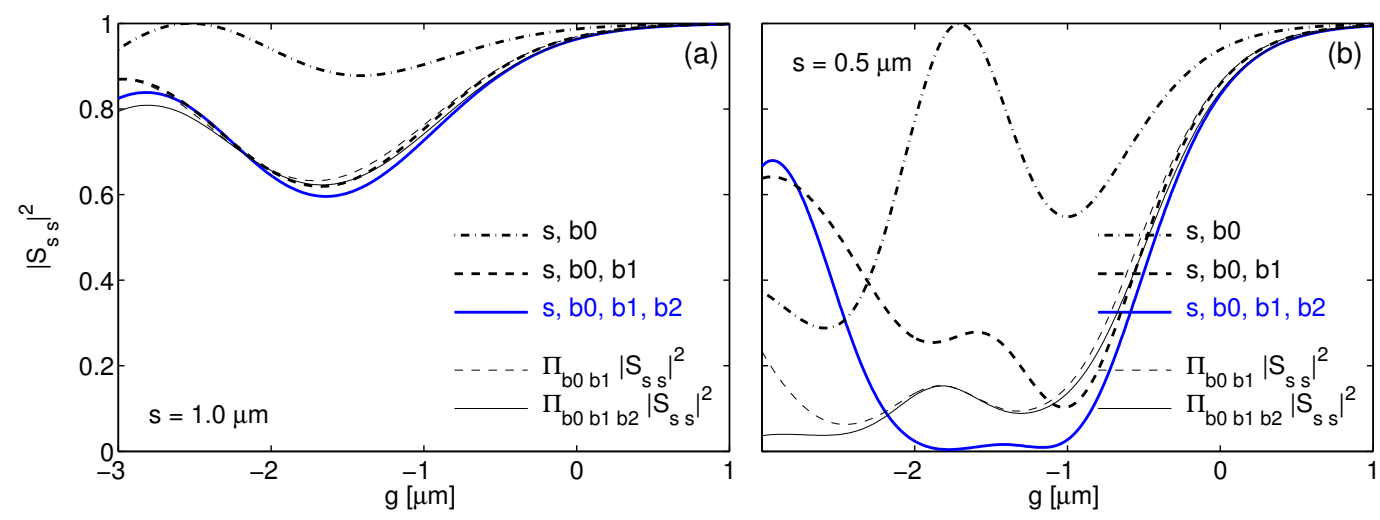

Figure 9: Straight-straight power coupling coefficients $\left|\mathrm{S}_{s}\right|^{2}$ for the structure of Figure 6 with vertical separations $s=1.0 \mu \mathrm{m}$ (a) and $s=0.5 \mu \mathrm{m}$ (b). Bold curves: Results of CMT calculations with different modal basis sets; the mode of the straight waveguides and the fundamental bend mode b0 (dash-dotted), s and the two lowest order bend fields b0, b1 (dashed), or s and the three lowest order bend fields b0, b1, b2 (continuous) are taken into account. Thin lines: Products of results for $\left|\mathrm{S}_{s s}\right|^{2}$ as computed in separate CMT calculations with mode s and only one of b0, b1, b2 (see the text for further details).

For the larger vertical separation (a), the CMT results s, b0 for one and s, b0, b1 for two disk modes differ considerably. Adding the third mode (curve s, b0, b1, b2) does not have as large an influence. It seems that the third mode just syphons off a fraction of the power of the straight waveguide, as can be seen also by noticing the small interaction levels of mode b2 with other fields in Figure 8 a $a, b, c)$. As to be expected, in case of the smaller vertical separation $s=0.5 \mu \mathrm{m}$ (b), the levels of exchanged power are in general much higher than for the larger separation. For gaps down to about $-1.0 \mu \mathrm{m}$ again a representation of the cavity field by the two fundamental disk modes seems to be sufficient, while for bus waveguide positions further underneath the disk the results change drastically, when the second order cavity mode is taken into account. Hence one should not trust the CMT results for $s=0.5 \mu \mathrm{m}$ and relative positions $g$ below about $-1.5 \mu \mathrm{m}$; just the fact that the third mode alters the results so significantly seems to indicate that more disk modes would be required for trustworthy CMT calculations. An intuitive argument why CMT would not be reliable in this range of core positions is found in the observation that the lower order cavity modes are predominantly located at the rim of the disk. The more the straight waveguide moves under the disk, the more basis modes are needed. Note that at 
certain $z$-positions for smaller values of $g$ the structure resembles a coupler consisting of a straight waveguide underneath an upper slab. Here one can no longer expect the representation of the complete field by the three disk modes to be correct. However, these regimes with rather strong interaction are not interesting for resonator applications anyway (cf. Section 3.2.2).

The thin curves in Figure 9 address the question, to what extent the bend modes can be considered independently. This amounts to a simplified model, where one regards the multimode disk as a sequence of cavities that interact with the same bus waveguides. Each cavity supports one of the modes b0, b1, or b2 and extracts a certain amount of power from the input channel. Then the throughput power $\left|S_{s s}\right|^{2}$ for the composite multimode device should be given by the product of the relative throughputs computed for the individual single-mode cavities. The lines $\prod_{b 0 \mathrm{~b} 1}\left|\mathrm{~S}_{\mathrm{ss}}\right|^{2}$ and $\prod_{\mathrm{b} 0 \mathrm{~b} 1 \mathrm{~b} 2}\left|\mathrm{~S}_{\mathrm{ss}}\right|^{2}$ in Figure 9 show these model results, where the values for $\left|\mathrm{S}_{\mathrm{s} s}\right|^{2}$ from separate CMT-calculations with bimodal basis sets $\{\mathrm{s}, \mathrm{b} j\}$, for $j=1,2$ and $j=1,2,3$, respectively, have been multiplied.

For the larger vertical separation (a), one observes a rather good agreement with the full CMT computations, corresponding to the only minor cross-coupling powers for the disk modes in Figure 8 (c). By allowing to skip the evaluation of the overlap integrals between the cavity fields, here the simplified model provides a much faster way of calculating the coupler scattering matrix. According to Figure $8(f)$, the presence of the bus core effects a much stronger interaction between the disk fields for the smaller separation $s=0.5 \mu \mathrm{m}$. In this case these coefficients can obviously not be neglected; the simplified viewpoint of independent cavity modes fails, as reflected by the pronounced disagreement of the thin and bold lines in Figure 9(b). A similar discussion for 2-D configurations can be found in Ref. [14], where the inherent 3-D effects of strong interaction due to the horizontally overlapping components could not be observed.

\subsubsection{Comparison with BPM simulations}

A comparison to entirely independent numerical simulations is meant as an additional validation of our CMT implementation. We apply a semi-vectorial Beam Propagation Method (BPM) that is part of a commercial software environment for integrated optics simulations [35]. Figure 10] shows a reassuring qualitative and also some quantitative agreement between the two methods.

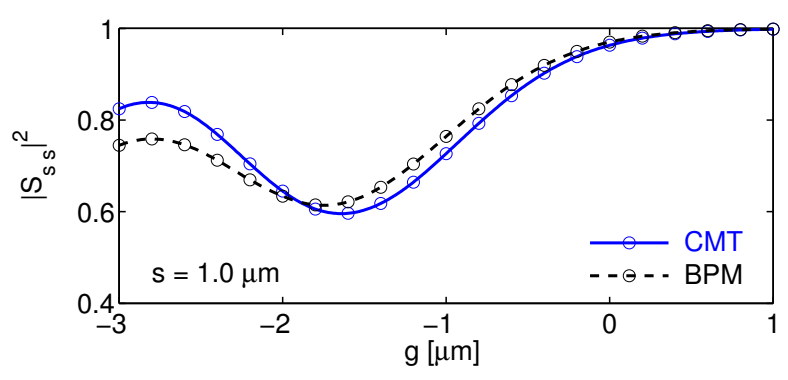

Figure 10: Self-coupling power $\left|\mathrm{S}_{s}\right|^{2}$ of the straight waveguide mode as computed by the present CMT approach (modes s, b0, b1, b2, continuous line) and by a commercial semivectorial BPM program [35] (dashed curve), for the structure of Figure 6 with a vertical separation $s=1.0 \mu \mathrm{m}$ and varying horizontal core position $g$.

The BPM version applied here does have notable shortcomings in this comparison: It is not fully vectorial, and does not contain any wide-angle corrections. This implies that in principle only the unidirectional light propagation along and close to a single axis is treated correctly (which should be of less importance, if one considers the throughput power is the bus waveguide only, since the disk takes the field away from the straight core before any relevant wave vector angles exceed the ranges that are well approximated by the BPM). While projections onto modes of straight waveguides are provided, it is not straightforward to extract the modal amplitudes of the (multiple) cavity modes from the calculated fields. This prohibits a discussion of roles of individual modes as in the paragraphs above, and the extension of the coupler simulations to predictions of multimode resonator spectra. Therefore, the comparison in Figure 10 is restricted to the throughput power in the straight waveguide.

\subsubsection{Alternative basis modes}

As discussed at the beginning of this section, for the computations of the cavity basis fields one may choose whether or not to treat the silicon dioxide substrate as part of the constituting permittivity profile (options (b) and (c) in Figure 6). So far all CMT results shown rely on basis modes as in Figure 7 that are defined for 
the disk structure including the substrate. Figure 11 compares the diagonal coefficients of the scattering matrix (part (d) of Figure 8), computed according to both options.

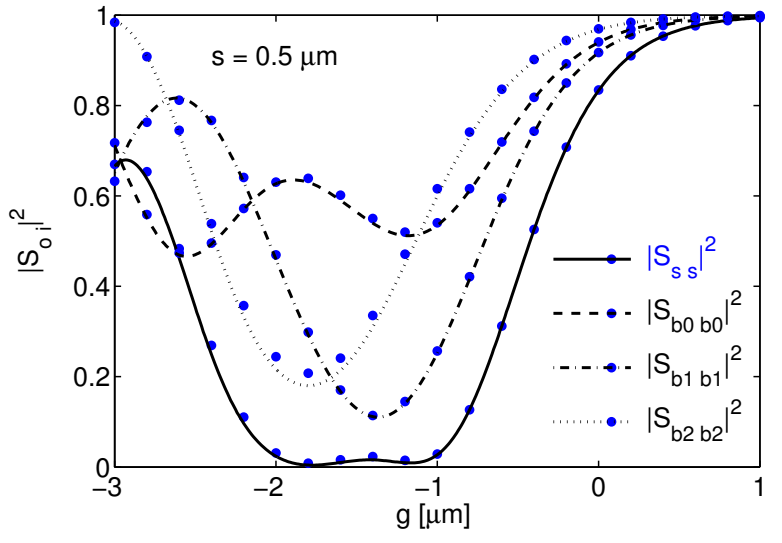

Figure 11: CMT computations with different sets of basis modes, related to the two options for a structure division that are sketched in Figure 6 The curves show the power self-coupling coefficients of the four basis modes versus the lateral position $g$ of the straight core, for a vertical separation $s=0.5 \mu \mathrm{m}$. Lines: the bend modes b0, b1, b2 of Figure 7 and Table 1, option (b) in Figure 6, marker symbols: the three lowest order bend modes supported by the disk core embedded in the cladding, for absent substrate, option (c) in Figure[

Only very minor differences appear for the present coupler device, even in the configurations for $s=0.5 \mu \mathrm{m}$ with strong interaction, where the changes are most pronounced for the second order cavity field $b 2$ that spreads furthest down into the substrate region. Most likely the agreement is due to the present rather low refractive index contrast between the substrate and cladding regions (the substrate hardly influences the profiles in Figure 7 . Still, in simulation of other structures we have observed that it can be essential to include a substrate and other infinite, rotational invariant slabs into the structures that the cavity modes are calculated for. A typical example could be a bus waveguide, that is prepared as a non-completely etched straight rib, where the remaining lateral film supports guided slab modes. In that case the cavity fields can spread out quite far radially into the slab below the cavity (leakage into the slab modes); these effects are likely to be overlooked if a priori the lower film layers are neglected when computing the cavity modes.

The CMT naturally incorporates any small phase changes of basis fields due to differences between the constituting and the complete permittivity profiles into the diagonal coefficients of the CMT equations (5), (6). Note that these phases, if non-negligible, need also to be taken into account when evaluating the corresponding resonator spectrum. Also then a definition of the constituting structure for the cavity mode computation as close as possible to the full device is desirable.

\subsubsection{Field examples}

Given the numerical solution of Eqs. (5), (6) and the mode profile data, the CMT model provides full information about the electromagnetic field in the coupler region. Figures 12, 13 illustrate the field in the structure for a disk / bus position of $s=0.5 \mu \mathrm{m}$ and $g=-1.0 \mu \mathrm{m}$.

Light is launched into the straight waveguide. At the vertical level of the bus core (a), the plot shows the depleting of the straight waveguide mode, while at a higher position inside the disk (b), one observes the beginning of the beating of the three cavity modes. The central cross section in Figure 13 exhibits a field distribution with similar field strengths in the disk and in the straight core. Note that for visualization purposes here a configuration with strong interaction has been selected, that transfers almost the entire input power from the straight waveguide to the disk.

In principle, the abstract CMT solutions ( $z$-evolution of the coupler transfer matrices) and the known field evolutions of the cavity modes, together with the consistent set of local amplitudes for the cavity loop, should also allow to generate field data for entire microresonator devices. The procedure is completely analogous to the 2-D case [14] (though not implemented so far), where here the 3-D, fully vectorial optical field would be accessible.

\subsubsection{Resonator spectra}

By evaluating the expressions in Ref. [14] for the power transfer through the symmetric, composite device with two couplers and the multimode cavity, the prediction of microresonator spectra associated with the structure of Figure 6 is straightforward. Figure 14 summarizes results for different relative positions of bus waveguides and 

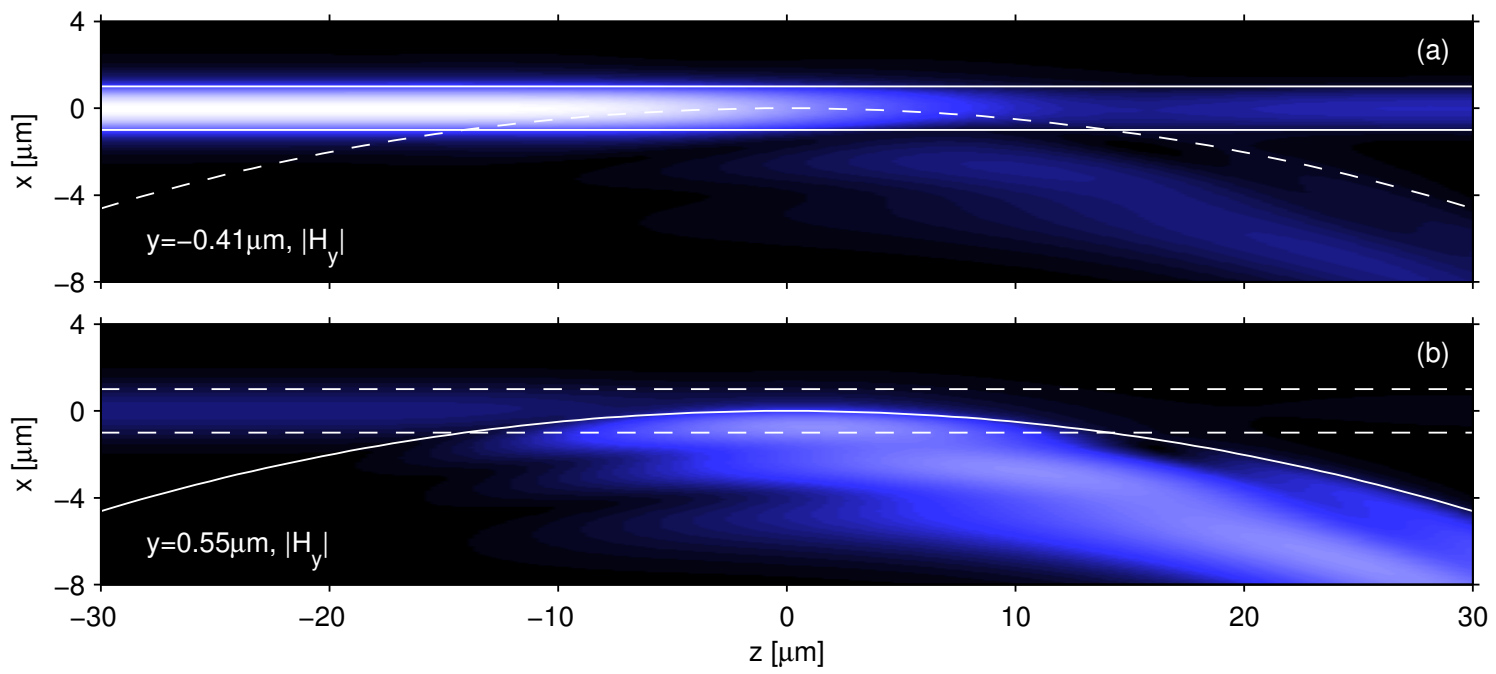

Figure 12: Coupled mode propagation through the coupling region as defined in Figure 6, for a vertical separation $s=0.5 \mu \mathrm{m}$ and horizontal position $g=-1.0 \mu \mathrm{m}$. The plots show the absolute value $\left|H_{y}\right|$ of the dominant magnetic component for the TE-like polarized fields. The guided mode of the straight waveguide is launched at $z_{i}=-30 \mu \mathrm{m}$. Horizontal field cross sections at $y=-0.41 \mu \mathrm{m}$, close to the center of the straight core (a), and at $y=0.55 \mu \mathrm{m}$, near the center of the disk (b).

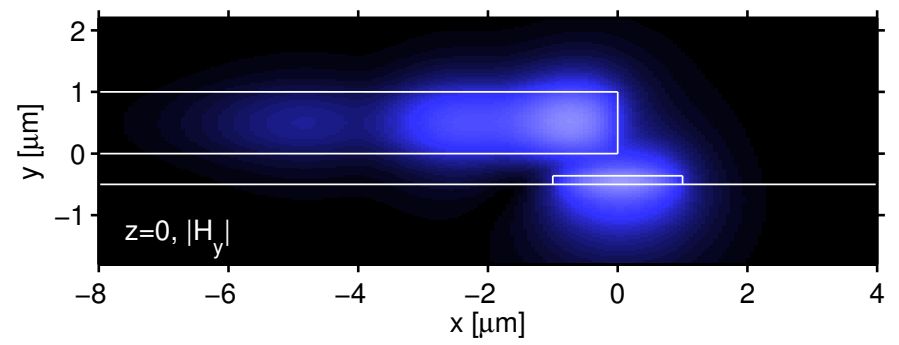

Figure 13: Vertical field cross section through the center $z=0$ of the coupler structure as defined in Figure 6 for a configuration as shown in Figure 12 The gray scales correspond to the dominant magnetic component $\left|H_{y}\right|$ of the TE-like polarized field; the levels are comparable with Figure 12

cavity, where the simplified procedure as outlined at the end of Section 2 is applied, for the target wavelength $\lambda=1.55 \mu \mathrm{m}$.

For the large bus-cavity separation (a) with moderate coupling strength one observes an array of three well separated, narrow peaks in the dropped power or dips in the transmitted power, respectively. The array repeats periodically when looking at larger or smaller wavelengths. Each of these three dips corresponds to one of the three disk modes: By inspecting the relative amplitudes of the individual modes in the cavity segments at the resonance wavelength, we can identify the mode that is relevant for the resonance. Counting from left to right, the first, broadest and least pronounced dip corresponds to the second order, most lossy disk mode b2. The second peak, in which the dropped power almost reaches $100 \%$, can be ascribed to the fundamental mode b0, while the last resonance is due to the first order field b1.

As expected, for smaller separations $(b, c)$ with stronger interaction between the bus waveguides and the cavity, the quality of the resonances decreases due to the losses to the straight waveguides. The peaks are shifted moderately, they broaden, become more pronounced, and start to overlap. Qualitatively similar changes with respect to (a) can be achieved either by shifting the bus cores towards the center of the cavity (b), or by reducing the vertical distance between bus cores and cavity (c).

Part (d) refers to a configuration with extreme interaction between the waves in the bus waveguides and the three cavity modes. According to Figures 8 (d, e) and 12] in an isolated coupler with separations $s=0.5 \mu \mathrm{m}$ and $g=-1 \mu \mathrm{m}$ almost the entire input power is extracted from the bus waveguide and distributed among the cavity fields. If the coupler is embedded in the resonator, for most wavelengths the reverse happens at the second coupler, and a large fraction of the input power is directly transferred to the drop port, in a non-resonant manner. The bus cores, however, effect also a strong interaction between the cavity modes; the resulting field pattern with the wavelength dependent relative phase relations of the disk modes then leads to the power transfer function of (d) with the seemingly reversed roles of transmitted and dropped power. Clearly, here one can no longer speak of individual resonances. 

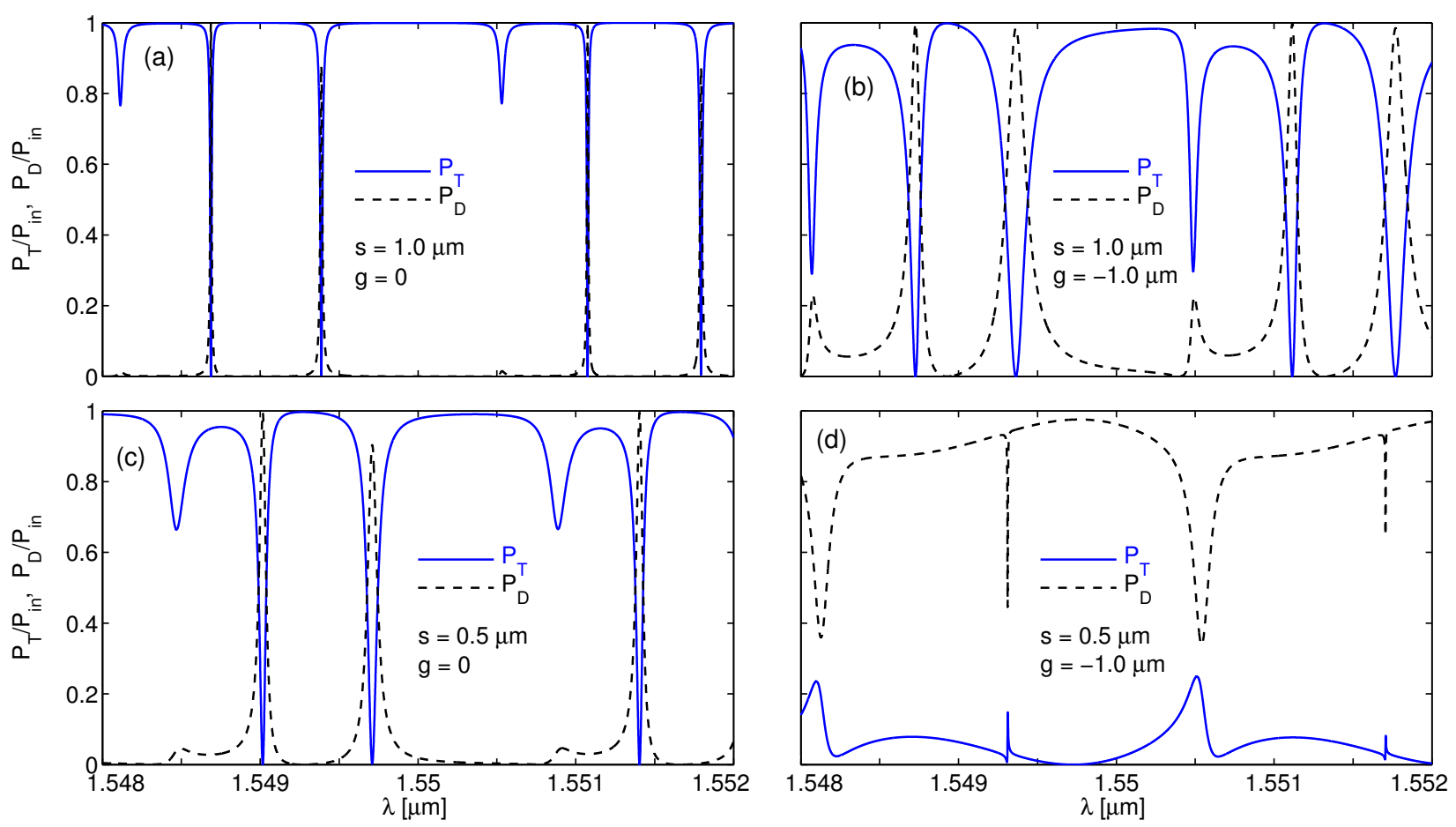

Figure 14: Relative dropped and transmitted power $P_{D}, P_{T}$ versus the vacuum wavelength of the input light, for microresonators that consist of two identical couplers according to Figure 6 with different vertical and horizontal separations $s$ and $g$ between the cavity and the bus cores.

\subsection{Hybrid ring and pedestal waveguide coupler}

For the last example we choose a resonator structure with the coupler cross section sketched in Figure [15] As before, the straight bus waveguides are created from silicon nitride, where here the supporting silicon dioxide layer is partially etched away isotropically, and replaced by the polymer cladding. Compared to the not-underetched structure, the present waveguides are closer to a vertically symmetric configuration: the mode profiles extend further upwards, and one can expect a higher coupling efficiency (this is a structure under fabrication at the moment). The geometry of the ring cavity was selected to explore the vectorial nature of the couplers, resembling e.g. an extreme example of a fabrication error. Due to the tilted outer flank, the ring core supports bend modes that are no longer nearly horizontally (TE) or vertically (TM) polarized, but are pronouncedly hybrid. Effects of polarization conversion can be expected for this resonator; cf. Refs. [36, 37] for corresponding theoretical and experimental results.

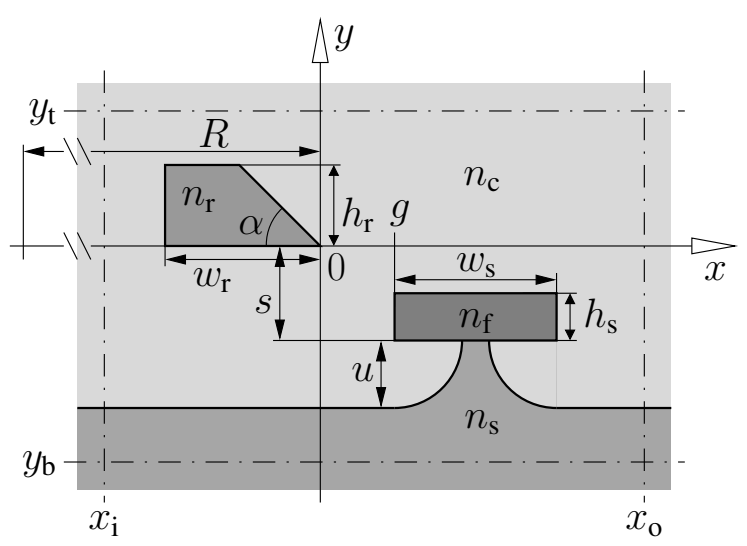

Figure 15: A hybrid coupler configuration. The $\mathrm{Si}_{3} \mathrm{~N}_{4}$ straight waveguide $\left(n_{f}=2.009\right)$ with dimensions $h_{s}=0.27 \mu \mathrm{m}$ and $w_{s}=2.5 \mu \mathrm{m}$ is supported by an isotropically underetched $\mathrm{SiO}_{2}$ pedestal $\left(n_{s}=1.45\right)$ with a depth $u=0.9 \mu \mathrm{m}$. The polymer ring with index $n_{r}=1.6275$, bottom width $w_{r}=2.0 \mu \mathrm{m}$, height $h_{r}=1.0 \mu \mathrm{m}$, and radius $R=100 \mu \mathrm{m}$ has an outer sidewall that is tilted at an angle $\alpha=48^{\circ}$. The vertical distance between the bottoms of the two cores is $s=1.0 \mu \mathrm{m} ; \mathrm{g}$ specifies the position of the left flank of the straight waveguide relative to the rim of the ring. A polymer cladding $\left(n_{c}=1.412\right) \mathrm{cov}$ ers the entire structure. Numerical parameters: $\Delta x=50 \mathrm{~nm}$, $\Delta y=27 \mathrm{~nm}, \Delta z=2.0 \mu \mathrm{m} ;\left[x_{i}, x_{o}\right]=[-12,4] \mu \mathrm{m},\left[y_{b}, y_{t}\right]=$ $[-3.7,4.4] \mu \mathrm{m},\left[z_{i}, z_{\mathrm{o}}\right]=[-35,35] \mu \mathrm{m}$.

Due to the inherent field representation, the FMM mode solver [17] applied for the calculation of the CMT basis modes is restricted to rectangular cross section geometries. Hence, for the present structure, the curved pedestal and the slanted sidewall of the ring are discretized using rectangles with a height of $0.1 \mu \mathrm{m}$. This approximation affects also the evaluation of the integrals in Eq. (5). 


\subsubsection{Coupler simulations}

Five basis fields enter the CMT description of this structure. The straight waveguide (remaining substrate, pedestal region, rectangular core, and homogeneous cladding) supports three guided modes, a fundamental and first order quasi-TE- and a fundamental quasi-TM-polarized field. These modes will be denoted by the symbols $\mathrm{S}-\mathrm{TE}_{0}, \mathrm{~S}-\mathrm{TE}_{1}$, and $\mathrm{S}-\mathrm{TM}$. The cavity ring (the ring core including the substrate, without pedestal) supports two bend modes with low attenuation, both of which are hybrid, i.e. both profiles exhibit non-negligible vertical and horizontal electric and magnetic field components. Symbols $\mathrm{R}_{0}$ and $\mathrm{R}_{1}$ identify these modes, where the polarization of the $\mathrm{R}_{0}$ field leans more towards TE, the mode $\mathrm{R}_{1}$ is closer to TM polarization. Table 2 summarizes the effective mode indices of these fields; Figure 16 illustrates the corresponding mode profiles.

\begin{tabular}{l|l} 
& $n_{\text {eff }}$ \\
\hline $\mathrm{S}^{\mathrm{TE}}$ & 1.625326 \\
$\mathrm{~S}-\mathrm{TE}_{1}$ & 1.553733 \\
$\mathrm{~S}-\mathrm{TM}$ & 1.511020 \\
$\mathrm{R}_{0}$ & $1.490975-\mathrm{i} 2.7 \cdot 10^{-7}$ \\
$\mathrm{R}_{1}$ & $1.483477-\mathrm{i} 1.2 \cdot 10^{-7}$
\end{tabular}

Table 2: Effective refractive indices $n_{\text {eff }}$ of the modes related to Figure 15 for a vacuum wavelength $\lambda=1.55 \mu \mathrm{m} ; S-T E_{0}, S-T E_{1}, S-T M_{0}$ : straight bus waveguide, $R_{0}, R_{1}$ : bend modes of the ring cavity. The values for the ring modes correspond to a definition of the bend radius $R$ as the outer rim of the ring, i.e. the modal fields $R_{0}$ and $R_{1}$ evolve with the angular coordinate $\theta$ in the cylindrical coordinate system of the ring as $\sim \exp \left(-\mathrm{i} k n_{\mathrm{eff}} R \theta\right)$.
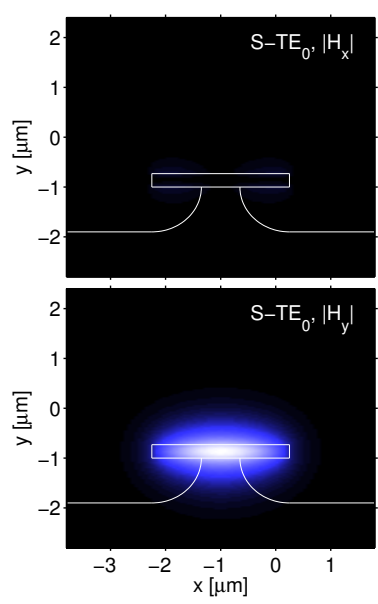
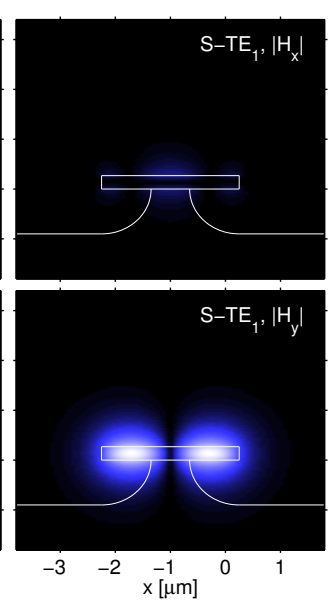
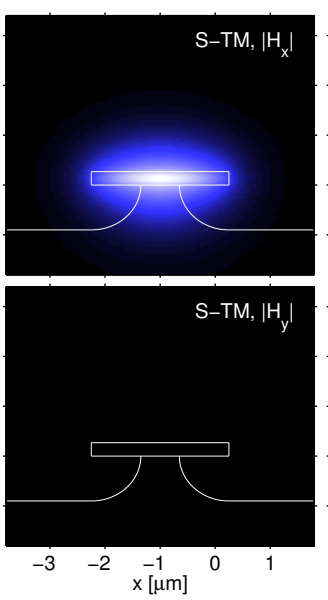
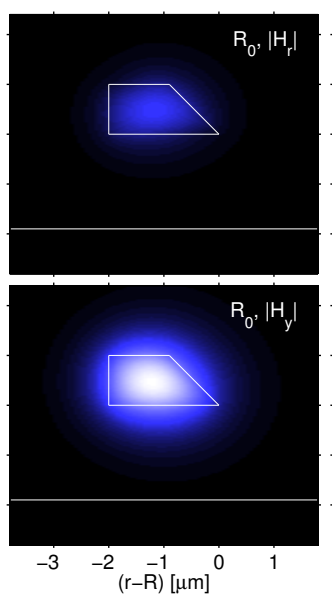
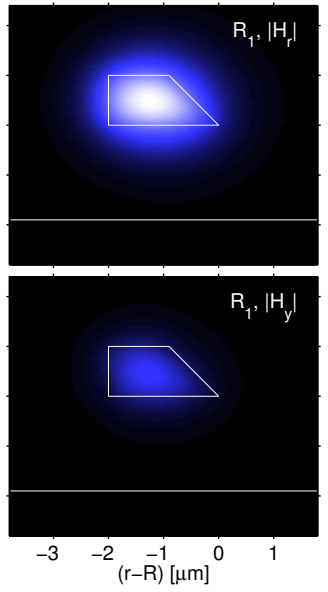

Figure 16: Vectorial mode profiles as applied for the coupled mode description of the structure of Figure 15 from left to right the three modes $S-T E_{0}, S-T E_{1}$, and $S$-TM of the straight waveguide, and the two hybrid ring modes $R_{0}$ and $R_{1}$. The gray scales correspond to the absolute values of the major horizontal $\left(\left|H_{x}\right|\right.$, or $\left|H_{r}\right|$, respectively, top) and the vertical magnetic field component $\left(\left|H_{y}\right|\right.$, bottom), where the levels in each column are comparable. Cf. the remark in the caption of Figure 7 on the role of the radial polar coordinate $r$. Uniform parameter settings for the FMM mode analysis computations: $M_{s}=200,\left[y_{b}^{M}, y_{t}^{M}\right]=[-5,3] \mu \mathrm{m}$.

The effective indices (real parts) of both ring modes are lower than the effective indices of the straight waveguide modes; especially the values of the two TE modes $\mathrm{S}-\mathrm{TE}_{0}$ and $\mathrm{S}-\mathrm{TE}_{1}$ are relatively far away from the levels of the ring modes $R_{0}$ and $R_{1}$. Besides the phase matching, according to Eq. (5) a strong interaction between two basis modes requires a large mode overlap, i.e. similarly polarized fields. One therefore expects that the modes $\mathrm{S}-\mathrm{TM}$ and $\mathrm{R}_{1}$ will exhibit the strongest interaction. However, due to the arbitrariness in the definition of the bend radius, and consequently in the effective mode indices for $R_{0}$ and $R_{1}$, the phase matching arguments should be used with some caution. For a gap of $-2.25 \mu \mathrm{m}$ (in the center plane $z=0$ of the coupler region the base of the ring is exactly centered above the straight waveguide), coupled mode calculations predict a coupler scattering matrix with absolute values as given in Table 3

\begin{tabular}{l|rrrrr}
$\swarrow$ & $\mathrm{S}^{-T_{0}}$ & $\mathrm{~S} \mathrm{TE}_{1}$ & $\mathrm{~S}-\mathrm{TM}$ & $\mathrm{R}_{0}$ & $\mathrm{R}_{1}$ \\
\hline $\mathrm{S}-\mathrm{TE}_{0}$ & 100.0 & $\approx 0$ & $\approx 0$ & $\approx 0$ & $\approx 0$ \\
$\mathrm{~S}-\mathrm{TE}_{1}$ & $\approx 0$ & 97.8 & $\approx 0$ & 1.9 & 0.1 \\
$\mathrm{~S}-\mathrm{TM}$ & $\approx 0$ & $\approx 0$ & 26.2 & 6.3 & 67.0 \\
$\mathrm{R}_{0}$ & $\approx 0$ & 2.1 & 6.7 & 86.2 & 5.5 \\
$\mathrm{R}_{1}$ & $\approx 0$ & 0.1 & 67.5 & 5.2 & 27.3
\end{tabular}

Table 3: Power scattering matrix of the coupler structure of Figure 15, for $g=-2.25 \mu \mathrm{m}$. The entry $\left|S_{o}\right|^{2}$ in row $o$ and column $i$ represents the relative power (in \%) transferred from input mode $i$ to output mode $o$. Entries " $\approx 0$ " are below the limit of accuracy that can be expected from the calculations. 
To some extend the results confirm our a priori expectation. The fundamental mode $\mathrm{S}-\mathrm{TE}_{0}$ of the straight waveguide propagates almost autonomously; also the first order TE mode S-TE ${ }_{1}$ interacts only weakly with the two ring modes. The largest off-diagonal coefficient reflects the strong coupling between the TM mode S-TM of the straight waveguide and the similarly polarized ring mode $\mathrm{R}_{1}$. One interesting and perhaps unexpected feature is the rather strong coupling between the two ring modes due to the presence of the straight core; about $5 \%$ of the power in $R_{0}$ is transfered to $R_{1}$, and vice versa.

Note that, despite the manifold approximations inherent in these simulations, the CMT results respect power conservation remarkably well (which holds only up to the losses due to the attenuation of the bend modes). The sums of the rows and columns of Table 3 deviate from unity by not more than $0.5 \%$. The same applies to reciprocity; corresponding off-diagonal entries differ by not more than this value. While this can be seen as some validation for the results, one can also regard this as an indication in how far the numerical values can be trusted.

Figure 17 provides an impression of the field evolution along the propagation axis, in case the coupler structure is excited by the mode S-TM of the bus waveguide. The power distribution shown here corresponds to the column S-TM in Table 3. During propagation, the power concentrated initially only in the TM field of the straight waveguide is transfered partly to the cavity core, but remains not restricted to TM $\left(H_{x}\right)$ polarization. In the exit plane of the coupler region, both hybrid modes of the ring cavity carry some power, i.e. both magnetic field components are present.
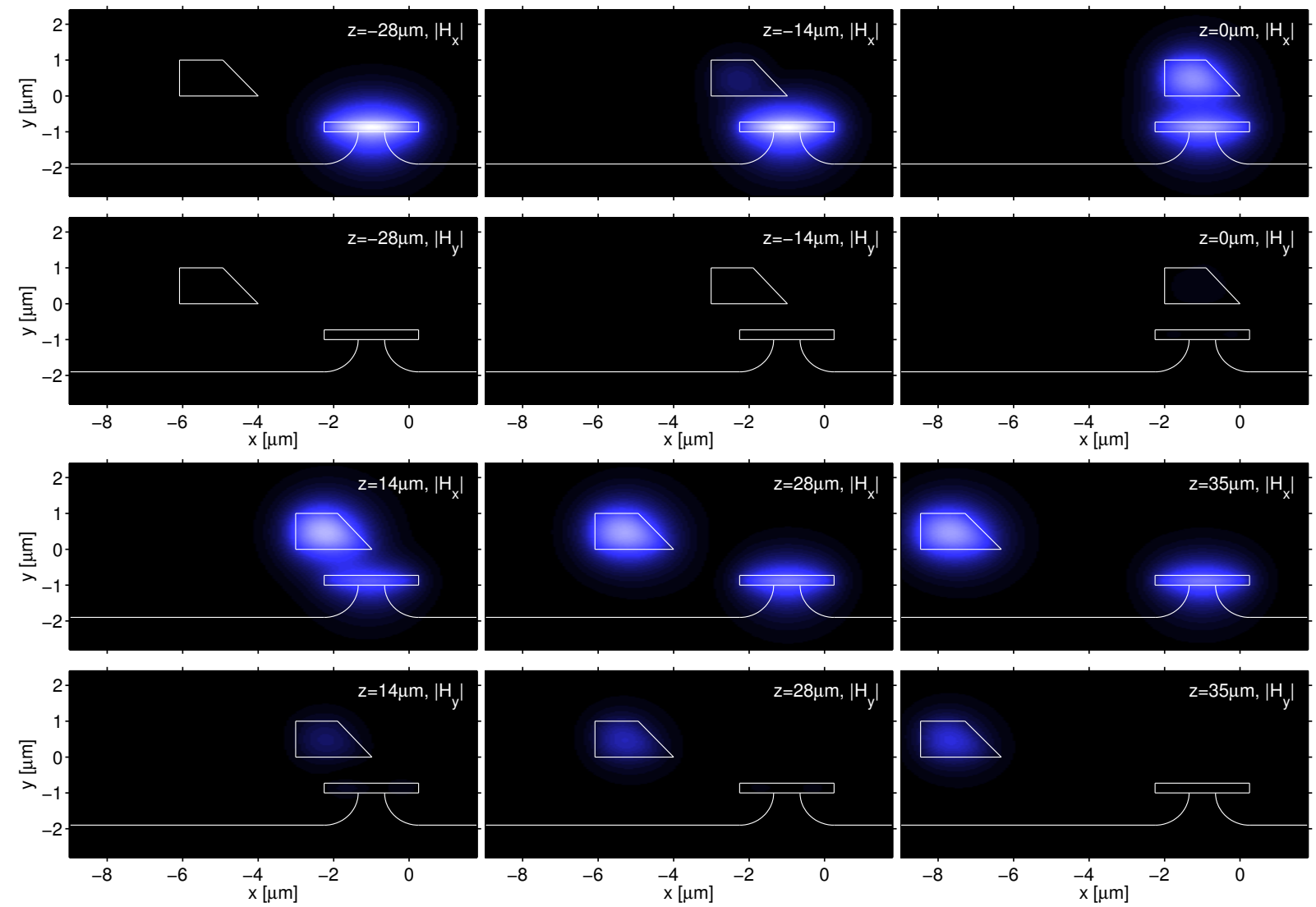

Figure 17: Transverse magnetic fields $\left(\left|H_{x}\right|\right.$, upper insets in each row, and $\left|H_{y}\right|$, below) on a series of vertical cross section planes at different $z$-positions for a coupler configuration according to Figure 15 with $g=-2.25 \mu \mathrm{m}$. Light is launched at $z_{i}=-35 \mu \mathrm{m}$ into the mode $S$-TM of the straight waveguide. The gray scales of all subplots are comparable.

Outside the coupler regions, the modes $\mathrm{R}_{0}$ and $\mathrm{R}_{1}$ propagate independently along the curved core, each with its own propagation constant. They establish an interference pattern with a half beat length given by the inverse difference of the effective indices (here $\lambda /\left(n_{\text {eff,R } 0}-n_{\text {eff,R } 1}\right) / 2=103 \mu \mathrm{m}$; the cavity length is $2 \pi R=628 \mu \mathrm{m}$ ), that can be viewed as periodic polarization conversion. It depends on the precise excitation conditions (relative amplitude and phase; for the full ring including the contributions from the incoming fields in both the bus and the cavity cores) whether the process reaches states with - up to the mismatch of the respective (complex) mode profile components - almost TM or TE polarization, where locally the vertical or radial magnetic field components cancel. 


\subsubsection{Ringresonator spectra}

The two bus waveguides of a microresonator consisting of coupler regions according to Figure [15] each support three guided modes. Hence, for the through and drop output channels, three modes ("ports") must be considered. According to Table 3 , the S-TE $\mathrm{TE}_{0}$ field does not play a role in the interaction; any input power in this mode will propagate straight on to the through port. We therefore restrict the further discussion to the throughput powers $P_{\mathrm{T}}^{\mathrm{TE} 1}, P_{\mathrm{T}}^{\mathrm{TM}}$, and the dropped powers $P_{\mathrm{D}}^{\mathrm{TE} 1}, P_{\mathrm{D}}^{\mathrm{TM}}$ in modes $\mathrm{S}-\mathrm{TE}_{1}$ and $\mathrm{S}-\mathrm{TM}$. Figure 18 a, b) shows the CMT results for the spectrum of the ring-resonator with $g=-2.25 \mu \mathrm{m}$, i.e. the same configuration as discussed in Section 3.3.1 An external excitation by the mode S-TM of the straight waveguide is considered.

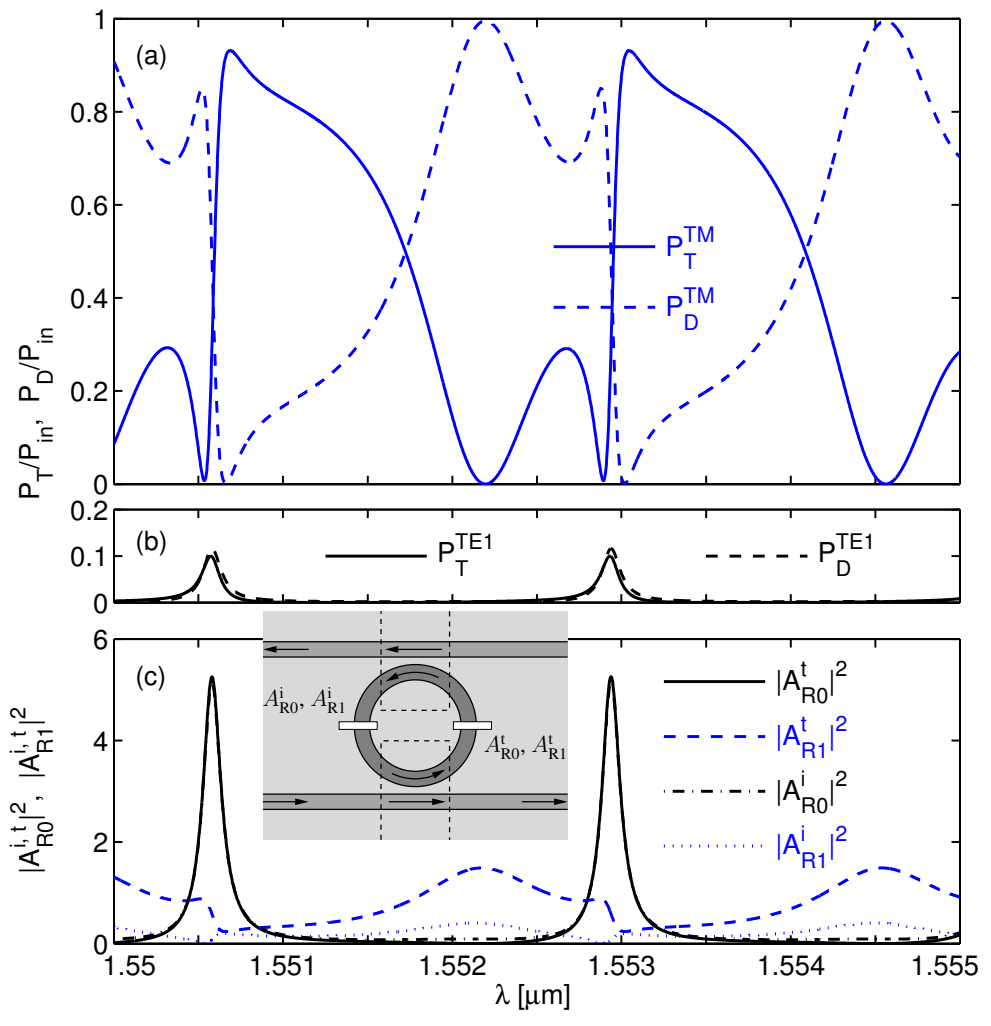

Figure 18: Spectrum of a symmetric resonator consisting of two couplers as specified in Figure 15 for an excitation by mode $S-T M$ in one of the bus waveguides. $(a, b)$ : Relative power transfered to the through $\left(P_{T}\right)$ and drop ports $\left(P_{D}\right)$ for the two relevant polarized modes (indices TE1 and TM for $S$ $T E_{1}$ and $\left.S-T M\right)$ supported by the bus waveguides. (c): Squared amplitudes of the two ring modes (subscripts $R 0$ and $R 1$ ) at the input (index i) and throughput side (index $t$ ) of the cavity. Note that this concerns amplitude levels of power-normalized modes, that establish upon excitation of the structure by a field with unit input power. The curves are thus directly comparable to the unit input amplitude.

Due to the multitude of modes present in this structure, the through and drop spectra contain some quite unexpected phenomena. For an interpretation of these, it will prove to be helpful to take a look at the local amplitudes of the two bend modes. For Figure 18 (c), the local amplitudes $A_{\mathrm{R} 0}^{\mathrm{i}}, A_{\mathrm{R} 1}^{\mathrm{i}}$ at the input side, and $A_{\mathrm{R} 0}^{\mathrm{t}}$, $A_{\mathrm{R} 1}^{\mathrm{t}}$ at the throughput side of the cavity ring have been evaluated as a function of the vacuum wavelength.

The most outstanding feature are the pronounced, relative sharp peaks in the amplitudes of the (more TE-like) mode $\mathrm{R}_{0}$, and the approximate equality of their absolute values on the input- and throughput side. As per Table 3, one would expect a drop of $13.8 \%$ in the squared amplitude as the $\mathrm{R}_{0}$ field passes the top coupler (where no external input is present). Apparently, some amount of power is transferred to $\mathrm{R}_{0}$ from the ring mode $\mathrm{R}_{1}$, with a phase that happens to be correct. In a resonance of Lorentzian form, which the shape of the $\mathrm{R}_{0}$ peak is very close to, the phase of the oscillator changes rapidly close to the amplitude maximum. This explains the sharp variation of the otherwise rather broad $R_{1}$ curves near the $R_{0}$ resonance: the amplitude of $R_{1}$ at the output sides of the couplers consists of contributions from both $R_{1}$ and $R_{0}$, and around the resonance the relative phase of the mode $\mathrm{R}_{0}$, and consequently also their interference, changes quickly with the wavelength. Perhaps one can alternatively view the features around the $R_{0}$ peaks as as a resonance of the full cavity ring, where both $\mathrm{R}_{0}$ and $\mathrm{R}_{1}$ participate.

Much simpler to explain, the mode $\mathrm{R}_{1}$ at its resonance (where the $\mathrm{R}_{0}$ amplitudes are insignificant) loses the expected amount of power; the squared amplitude $A_{\mathrm{R} 1}^{\mathrm{i}}$ at the input side is about $72.8 \%$ lower than the amplitude square $A_{\mathrm{R} 1}^{\mathrm{t}}$ on the throughput side. Due to the large amount of power that is lost at each coupler, the $\mathrm{R}_{1}$ resonance is of low quality, i.e. of a very broad shape.

Now looking again at the output spectrum (b), one finds that almost the same amounts $P_{\mathrm{T}}^{\mathrm{TE} 1}$ and $P_{\mathrm{D}}^{\mathrm{TE} 1}$ of power are dropped and directly transmitted into TE polarized guided waves. This can be explained by inspecting 
Figure 18 (c) and Table 33, only the ring mode $R_{0}$ couples significantly to $S-T E_{1}$, hence the shape of the $R_{0}$-peak in (c) corresponds directly to the peaks in $P_{\mathrm{T}}^{\mathrm{TE} 1}$ and $P_{\mathrm{D}}^{\mathrm{TE} 1}$ in (b).

According to Table 3, the straight waveguide mode S-TM interacts with both ring modes, though much more significantly with $R_{1}$. However, at resonance the power in $R_{0}$ is relatively large, and it can influence the $S$ TM output significantly in these wavelength ranges. This is indeed what we see; the main shape of both the through and the drop curves $P_{\mathrm{T}}^{\mathrm{TM}}$ and $P_{\mathrm{D}}^{\mathrm{TM}}$ is the broad response due to the ring mode $\mathrm{R}_{1}$, modulated near the $\mathrm{R}_{0}$ resonance. The drop response $P_{\mathrm{D}}^{\mathrm{TM}}$ mainly follows the $A_{\mathrm{R} 1}^{\mathrm{t}}$ curve from Figure 18(c), though it falls much deeper on the righthand side of the $\mathrm{R}_{0}$ resonance; the values very nearly reach zero. This must be caused by the interaction with the $\mathrm{R}_{0}$ ring mode, either as a coincidental feature, or due to some mechanism that we are not aware of (in principle, all is hidden in the 16 complex coefficients of the coupler scattering matrix, and the two complex propagation constants of the cavity modes). Concerning the through response $P_{\mathrm{T}}^{\mathrm{TM}}$, one expects the zero level at the $\mathrm{R}_{1}$ resonance, since only $\mathrm{R}_{1}$ is present with non-negligible amplitude in this wavelength range. The near-to zero level in $P_{\mathrm{T}}^{\mathrm{TM}}$ just to the left of the $\mathrm{R}_{0}$ resonance also seems coincidental, caused as it is by a complex interplay of three modes, but might also be explainable.

\section{Conclusions}

An important task in the design of circular microresonators is the calculation of the scattering matrices of the couplers that connect the cavity internally and externally. We have presented a fully-vectorial 3-D frequency domain coupled mode theory formulation that provides these matrices in a fast and accurate way. Presuming the waveguide modes are precalculated, the speed of our (non-optimized) fully vectorial CMT implementation is comparable to a commercial semivectorial beam-propagation package. But in contrast to the BPM, the CMT formulation yields directly explicit values for all coefficients in the scattering matrices of the couplers, that allow to interpret the role of the individual bend and straight modes in the interaction between the cavity and the bus waveguides, as a basis for the subsequent resonator design. While the present implementation covers quite general structures composed of codirectional straight and circularly bent waveguides or curved interfaces, in principle also field solutions related to other, rather arbitrary permittivity profiles could serve as basis fields, as long as the field in the composite structure is adequately represented by the basis elements.

Within the underlying assumptions on the microresonator model (e.g. the neglect of reflections), accurate results can be expected for configurations where the CMT basis sets constitute adequate representations of the optical field in the coupler structure. The present examples and analogous 2-D calculations [14] show that the approach can master a (surprisingly) large range of problems, what concerns radiative basis fields and levels of refractive index contrast. Simulations of a multimode resonator with a disk-shaped cavity showed that a few whispering gallery modes are sufficient for a proper estimation of the resonator spectrum, as long as the bus waveguides are positioned outside or close to the rim of the disk. Here the approach allows to easily investigate a wide range of geometrical design parameters, for 3-D resonator structures with horizontal or vertical coupling.

For cases with low interaction strength we found that the interaction between the individual cavity modes due to the presence of the bus waveguides remains rather weak; one may view the multimode coupler as a concatenation of coupler segments, where in each coupler only one bend mode extracts power from the ongoing straight waveguide mode. By neglecting the cross-coupling coefficients between the cavity modes, i.e. by executing separate calculations with one bend field at a time, this observation allows to speed up the calculations considerably. Simulations of a structure that supports strongly hybrid ring modes (i.e. modes that are not nearly horizontally or vertically polarized) show that polarization effects may be important for the spectral response of the resonator. Still, the analytic information that is directly available in the coupled mode model is a key to an interpretation of the interesting and quite unexpected features in the spectrum.

\section{Acknowledgments}

This work has been supported by the European Commission (project IST-2000-28018, 'NAIS'). The authors thank E. van Groesen, H. J. W. M. Hoekstra, and the colleagues in the NAIS project for many fruitful discussions. 


\section{References}

[1] B. E. Little, S. T. Chu, H. A. Haus, J. Foresi, and J.-P. Laine. Microring resonator channel dropping filters. Journal of Lightwave Technology, 15(6):998-1005, 1997.

[2] M. K. Chin and S. T. Ho. Design and modeling of waveguide-coupled single-mode microring resonators. Journal of Lightwave Technology, 16(8):1433-1446, 1997.

[3] B. E. Little, S. T. Chu, W. Pan, and Y. Kokubun. Microring resonator arrays for VLSI photonics. IEEE Photonics Technology Letters, 12(3):323-325, 2000.

[4] Next-generation active integrated optic subsystems, 2001-2004. Information society technologies programme of the European Commission, project IST-2000-28018,

http://www.mesaplus.utwente.nl/nais/.

[5] R. Grover, V. Van, T. A. Ibrahim, P. P. Absil, L. C. Calhoun, F. G. Johnson, J. V. Hryniewicz, and P.-T. Ho. Parallelcascaded semiconductor microring resonators for high-order and wide-FSR filters. Journal of Lightwave Technology, 20(5):900-905, 2002.

[6] J. V. Hryniewicz, P. P. Absil, B. E. Little, R. A. Wilson, and P.-T. Ho. Higher order filter response in coupled microring resonators. IEEE Photonics Technology Letters, 12(3):320-322, 2000.

[7] M. Bertolotti, A. Driessen, and F. Michelotti, editors. Microresonators as building blocks for VLSI photonics, volume 709 of AIP conference proceedings. American Istitute of Physics, Melville, New York, 2004.

[8] D. J. W. Klunder, F. S. Tan, T. van der Veen, H. F. Bulthuis, G. Sengo, B. Docter, H. J. W. M. Hoekstra, and A. Driessen. Experimental and numerical study of SiON microresonators with air and polymer cladding. Journal of Lightwave Technology, 21(4):1099-1110, 2003.

[9] B. E. Little, S. T. Chu, W. Pan, D. Ripin, T. Kaneko, Y. Kokubun, and E. Ippen. Vertically coupled glass microring resonator channel dropping filters. IEEE Photonics Technology Letters, 11(2):215-217, 1999.

[10] D. J. W. Klunder, E. Krioukov, F. S. Tan, T. van der Veen, H. F. Bulthuis, G. Sengo, C. Otto, H. J. W. M. Hoekstra, and A. Driessen. Vertically and laterally waveguide-coupled cylindrical microresonators in $\mathrm{Si}_{3} \mathrm{~N}_{4}$ on $\mathrm{SiO}_{2}$ technology. Applied Physics B, 73:603-608, 2001.

[11] A. Taflove and S. C. Hagness. Computational Electrodynamis: The Finite Difference Time Domain Method, 2nd ed. Artech House, Norwood, MA, USA, 2000.

[12] F. Schmidt. Computation of discrete transparent boundary conditions for the 2D Helmholtz equation. Optical and Quantum Electronics, 30(5/6):427-441, 1998.

[13] M. Hammer. Quadridirectional eigenmode expansion scheme for 2-D modeling of wave propagation in integrated optics. Optics Communications, 235(4-6):285-303, 2004.

[14] K. R. Hiremath, R. Stoffer, and M. Hammer. Modeling of circular integrated optical microresonators by 2-D frequency-domain coupled mode theory. Optics Communications, 2005. (submitted).

[15] K. Mertens, B. Scholl, and H. J. Schmitt. New highly efficient polarization converters based on hybrid supermodes. Journal of Lightwave Technology, 13(10):2087-2092, 1995.

[16] M. Lohmeyer. Vectorial wave-matching mode analysis of integrated optical waveguides. Optical and Quantum Electronics, 30:385-396, 1998.

[17] L. Prkna, M. Hubálek, and J. Čtyroký. Field modelling of circular microresonators by film mode matching. IEEE Journal of Selected Topics in Quantum Electronics, 11(1):217-223, 2005.

[18] D. G. Hall and B. J. Thompson, editors. Selected Papers on Coupled-Mode Theory in Guided-Wave Optics, volume MS 84 of SPIE Milestone Series. SPIE Optical Engineering Press, Bellingham, Washington USA, 1993.

[19] C. Vassallo. Optical Waveguide Concepts. Elsevier, Amsterdam, 1991.

[20] D. Marcuse. Theory of Dielectrical Optical Waveguides, 2nd edition. Academic Press, London, 1991.

[21] K. Okamoto. Fundamentals of Optical Waveguides. Academic Press, SanDiego, 2000.

[22] D. R. Rowland and J. D. Love. Evanescent wave coupling of whispering gallery modes of a dielectric cylinder. IEE Proceedings, Pt. J, 140(3):177-188, 1993.

[23] B. E. Little, J.-P. Laine, and H. A. Haus. Analytic theory of coupling from tapered fibers and half-blocks into microsphere resonators. Journal of Lightwave Technology, 17(4):704-715, 1999.

[24] J. A. Loaiza, D. J. W. Klunder, H. F. Bulthuis, M. Lohmeyer, Hoekstra H. J. W. M., and A. Driessen. Application of the coupled mode theory to the problem of coupling between bent and straight waveguides. IEEE/LEOS Benelux Chapter, Proceedings of the 5th Annual Symposium, Delft, 215-218, 2000. 
[25] A. Stumpf, J. Kunde, U. Gubler, A.-C. Pliska-Leduff, and C. Bosshard. A study on a microring structure for practical devices. Optical and Quantum Electronics, 35:1205-1213, 2003.

[26] S. L. Chuang. A coupled mode formulation by reciprocity and a variational principle. Journal of Lightwave Technology, 5(1):5-15, 1987.

[27] M. Lohmeyer, N. Bahlmann, O. Zhuromskyy, and P. Hertel. Radiatively coupled waveguide polarization splitter simulated by wave-matching based coupled mode theory. Optical and Quantum Electronics, 31:877-891, 1999.

[28] L. Prkna. Rotationally symmetric resonant devices in integrated optics. Faculty of Mathematics and Physics, Charles University, Prague, and Institute of Radio Engineering and Electronics, Academy of Sciences of the Czech Republic, Prague, Czech Republic, 2004. Ph.D. Thesis.

[29] L. Prkna, M. Hubálek, and J. Čtyroký. Vectorial eigenmode solver for bent waveguides based on mode matching. IEEE Photonics Technology Letters, 16(9):2057-2059, 2004.

[30] E. A. J. Marcatili. Bends in optical dielectric guides. The Bell System Technical Journal, September:2103-2132, 1969.

[31] M. Hammer, K. R. Hiremath, and R. Stoffer. Analytical approaches to the description of optical microresonator devices. In M. Bertolotti, A. Driessen, and F. Michelotti, editors, Microresonators as building blocks for VLSI photonics, volume 709 of AIP conference proceedings, pages 48-71. American Institute of Physics, Melville, New York, 2004. Proceedings of the International School of Quantum Electronics, 39th course, Erice, Sicily (October 2003).

[32] K. R. Hiremath, M. Hammer, R. Stoffer, L. Prkna, and J. Čtyroký. Analytical approach to dielectric optical bent slab waveguides. Optical and Quantum Electronics, 37(1-3):37-61, 2005.

[33] R. Stoffer, K. R. Hiremath, and M. Hammer. Comparison of coupled mode theory and FDTD simulations of coupling between bent and straight optical waveguides. In M. Bertolotti, A. Driessen, and F. Michelotti, editors, Microresonators as building blocks for VLSI photonics, volume 709 of AIP conference proceedings, pages 366377. American Institute of Physics, Melville, New York, 2004. Proceedings of the International School of Quantum Electronics, 39th course, Erice, Sicily (October 2003).

[34] W. H. Press, S. A. Teukolsky, W. T. Vetterling, and B. P. Flannery. Numerical Recipes in C, 2nd ed. Cambridge University Press, 1992.

[35] OlympIOs Integrated Optics Software. C2V, P.O. Box 318, 7500 AH Enschede, The Netherlands; http://www.c2v.nl/software/ .

[36] B. E. Little and S. T. Chu. Theory of polarization rotation and conversion in vertically coupled microresonators. IEEE Photonics Technology Letters, 12(4):401-403, 2000.

[37] A. Melloni, F. Morichetti, and M. Martinelli. Polarization conversion in ring resonator phase shifters. Optics Letters, 29(23):2785-2787, 2004. 